This item was submitted to Loughborough's Research Repository by the author.

Items in Figshare are protected by copyright, with all rights reserved, unless otherwise indicated.

\title{
On the classification of discrete Hirota-type equations in 3D
}

PLEASE CITE THE PUBLISHED VERSION

http://dx.doi.org/10.1093/imrn/rnu086

\section{PUBLISHER}

(c) The Authors. Published by Oxford University Press.

\section{VERSION}

AM (Accepted Manuscript)

\section{PUBLISHER STATEMENT}

This work is made available according to the conditions of the Creative Commons Attribution-NonCommercialNoDerivatives 4.0 International (CC BY-NC-ND 4.0) licence. Full details of this licence are available at: https://creativecommons.org/licenses/by-nc-nd/4.0/

\section{LICENCE}

CC BY-NC-ND 4.0

\section{REPOSITORY RECORD}

Ferapontov, E.V., V.S. Novikov, and Ilia Roustemoglou. 2019. "On the Classification of Discrete Hirota-type Equations in 3D". figshare. https://hdl.handle.net/2134/20230. 


\title{
On the classification of discrete Hirota-type equations in 3D
}

\author{
E.V. Ferapontov, V.S. Novikov, I. Roustemoglou \\ Department of Mathematical Sciences \\ Loughborough University \\ Loughborough, Leicestershire LE11 3TU \\ United Kingdom \\ e-mails: \\ E.V.Ferapontov@lboro.ac.uk \\ V.Novikov@lboro.ac.uk \\ I. Roustemoglou@lboro.ac.uk
}

\begin{abstract}
In the series of recent publications $[15,16,18,21]$ we have proposed a novel approach to the classification of integrable differential/difference equations in 3D based on the requirement that hydrodynamic reductions of the corresponding dispersionless limits are 'inherited' by the dispersive equations. In this paper we extend this to the fully discrete case. Based on the method of deformations of hydrodynamic reductions, we classify 3D discrete integrable Hirota-type equations within various particularly interesting subclasses. Our method can be viewed as an alternative to the conventional multi-dimensional consistency approach.
\end{abstract}

MSC: 35Q51, 37K10.

Keywords: discrete integrable systems in 3D, dispersionless limit, hydrodynamic reductions, Hirota-type equations. 


\section{Introduction}

This paper is based on the observation that various forms of the 3D Hirota difference equation [20] can be obtained as 'naive' discretisations of second order quasilinear PDEs, by simply replacing partial derivatives $\partial$ by discrete derivatives $\triangle$. Although this recipe should by no means preserve the integrability in general, it does apply to a whole range of interesting examples. Thus, the dispersionless PDE

$$
\left(u_{1}-u_{2}\right) u_{12}+\left(u_{3}-u_{1}\right) u_{13}+\left(u_{2}-u_{3}\right) u_{23}=0,
$$

gives rise to the lattice $\mathrm{KP}$ equation [10, 36, 35],

$$
\left(\triangle_{1} u-\triangle_{2} u\right) \triangle_{12} u+\left(\triangle_{3} u-\triangle_{1} u\right) \triangle_{13} u+\left(\triangle_{2} u-\triangle_{3} u\right) \triangle_{23} u=0 .
$$

Similarly, the dispersionless PDE

$$
\partial_{1}\left(\ln \frac{u_{3}}{u_{2}}\right)+\partial_{2}\left(\ln \frac{u_{1}}{u_{3}}\right)+\partial_{3}\left(\ln \frac{u_{2}}{u_{1}}\right)=0,
$$

results in the Schwarzian KP equation [36, 13, 6, 7, 27],

$$
\triangle_{1}\left(\ln \frac{\triangle_{3} u}{\triangle_{2} u}\right)+\triangle_{2}\left(\ln \frac{\triangle_{1} u}{\triangle_{3} u}\right)+\triangle_{3}\left(\ln \frac{\triangle_{2} u}{\triangle_{1} u}\right)=0 .
$$

Here $u\left(x^{1}, x^{2}, x^{3}\right)$ is a function of three (continuous) variables. We use subscripts for partial derivatives of $u$ with respect to the independent variables $x^{i}: u_{i}=u_{x^{i}}, u_{i j}=u_{x^{i} x^{j}}, \partial_{i}=\partial_{x^{i}}$, etc. Forward/backward $\epsilon$-shifts and discrete derivatives in $x^{i}$-direction are denoted $T_{i}, T_{\bar{i}}$ and $\triangle_{i}, \triangle_{\bar{i}}$, respectively: $\triangle_{i}=\frac{T_{i}-1}{\epsilon}, \triangle_{\bar{i}}=\frac{1-T_{\bar{i}}}{\epsilon}$. We also use multi-index notation for multiple shifts/derivatives: $T_{i j}=T_{i} T_{j}, \triangle_{i \bar{j}}=\triangle_{i} \triangle_{\bar{j}}$, etc.

Our first main result (Theorem 1 of Sect. 3) provides a classification of integrable discrete conservative equations of the form

$$
\triangle_{1} f+\triangle_{2} g+\triangle_{3} h=0
$$

where $f, g, h$ are functions of $\triangle_{1} u, \triangle_{2} u, \triangle_{3} u$ only. Equations of this type appear as $\triangle$-forms of various discrete equations of the KP, Toda and Sine-Gordon type, see Appendix for examples and references. The corresponding dispersionless limits are scalar conservation laws of the form

$$
\partial_{1} f\left(u_{1}, u_{2}, u_{3}\right)+\partial_{2} g\left(u_{1}, u_{2}, u_{3}\right)+\partial_{3} h\left(u_{1}, u_{2}, u_{3}\right)=0 .
$$

Our approach to the classification of discrete integrable equations is based on the requirement that all hydrodynamic reductions of the corresponding dispersionless limit are inherited by the full discrete (dispersive) equation. This method has been successfully applied recently to various classes of differential/difference equations in $3 \mathrm{D}$, see $[15,16,18,21]$. A brief summary of the method is included in Sect. 2.

The classification is performed modulo elementary transformations $u \rightarrow \alpha u+\alpha_{i} x^{i}$, as well as permutations of the independent variables $x^{i}$, which preserve the class of discrete conservation laws (3).

We show that any integrable equation of the form (3) arises as a conservation law of a certain discrete integrable equation of octahedron type,

$$
F\left(T_{1} u, T_{2} u, T_{3} u, T_{12} u, T_{13} u, T_{23} u\right)=0,
$$


see [3] for their classification. More precisely, there exist seven cases of integrable octahedrontype equations (note that our equivalence group is different from the group of admissible transformations utilised in [3]), each of them possessing exactly three first-order linearly independent conservation laws of the form (3). Let $I, J, K$ denote their left hand sides. They give rise to a three-parameter family of integrable equations of the form (3),

$$
\alpha I+\beta J+\gamma K=0,
$$

where $\alpha, \beta, \gamma$ are arbitrary constants (see Theorem 1 for a complete list and explicit formulae); we prove that all integrable discrete conservative equations of the form (3) can be obtained by this construction. Thus, there exist seven three-parameter families of integrable conservation laws (3). One of these cases is associated with the octahedron equation

$$
\left(T_{2} \triangle_{1} u\right)\left(T_{3} \triangle_{2} u\right)\left(T_{1} \triangle_{3} u\right)=\left(T_{2} \triangle_{3} u\right)\left(T_{3} \triangle_{1} u\right)\left(T_{1} \triangle_{2} u\right)
$$

known as the Schwarzian KP equation in its standard form. It possesses three conservation laws

$$
\begin{aligned}
& I=\triangle_{2} \ln \left(1-\frac{\triangle_{3} u}{\triangle_{1} u}\right)-\triangle_{3} \ln \left(\frac{\triangle_{2} u}{\triangle_{1} u}-1\right)=0, \\
& J=\triangle_{3} \ln \left(1-\frac{\triangle_{1} u}{\triangle_{2} u}\right)-\triangle_{1} \ln \left(\frac{\triangle_{3} u}{\triangle_{2} u}-1\right)=0, \\
& K=\triangle_{1} \ln \left(1-\frac{\triangle_{2} u}{\triangle_{3} u}\right)-\triangle_{2} \ln \left(\frac{\triangle_{1} u}{\triangle_{3} u}-1\right)=0,
\end{aligned}
$$

note that their linear combination $I+J+K=0$ coincides with (2).

Our second result (Theorem 3 of Sect. 4) is the classification of discrete integrable quasilinear equations of the form

$$
\sum_{i, j=1}^{3} f_{i j} \triangle_{i j} u=0,
$$

where $f_{i j}$ are functions of $\triangle_{1} u, \triangle_{2} u, \triangle_{3} u$ only. These equations can be viewed as discretisations of second-order quasilinear PDEs

$$
\sum_{i, j=1}^{3} f_{i j} u_{i j}=0
$$

studied in [9]. In contrast to the result of Theorem 1, there exists a unique integrable example within this class, namely the lattice KP equation (1).

We also classify differential-difference degenerations of the above equations with one/two discrete variables (subsections 3.1, 3.2 and 4.1, 4.2). Some of the examples from Sect. 3.1 are apparently new.

In Sect. 5 we present the results of numerical simulations for the gauge-invariant form of the Hirota equation, exhibiting the formation of a dispersive shock wave.

In the Appendix we bring together $\triangle$-forms of various discrete $\mathrm{KP} /$ Toda type equations.

Our approach to the classification of discrete integrable equations in 3D can be viewed as an alternative to the conventional multi-dimensional consistency approach [5, 38], that has recently been extended to 3D equations [3]. Both methods have their advantages and limitations. Thus, the method of multi-dimensional consistency has so far been restricted to the class of 3D 
equations satisfying the additional octahedron property. On the other hand, our approach requires the existence of a non-degenerate dispersionless limit. It is not surprising however that both methods (even when applied to seemingly different classes of equations) lead to similar classification results: this reflects the universality of 3D Hirota-type equations.

\section{Preliminaries: the method of dispersive deformations}

This method applies to 3D dispersive equations possessing a non-degenerate dispersionless limit, and is based on the requirement that all hydrodynamic reductions of the dispersionless limit are 'inherited' by the full dispersive (in particular, difference) equation, at least to some finite order in the deformation parameter $\epsilon$, see $[15,16,18,21]$ for examples and applications. It turns out that all known integrable differential/difference equations in 3D pass this test. Our experience suggests that in most cases it is sufficient to perform calculations up to the order $\epsilon^{2}$, the necessary conditions for integrability obtained at this stage usually prove to be sufficient, and imply the existence of conventional Lax pairs, etc. Let us illustrate our approach by classifying integrable discrete wave-type equations,

$$
\triangle_{t \bar{t}} u-\triangle_{x \bar{x}} f(u)-\triangle_{y \bar{y}} g(u)=0,
$$

where $f$ and $g$ are functions to be determined. Using expansions of the form

$$
\triangle_{t \bar{t}}=\frac{\left(e^{\epsilon \partial_{t}}-1\right)\left(1-e^{-\epsilon \partial_{t}}\right)}{\epsilon^{2}}=\partial_{t}^{2}+\frac{\epsilon^{2}}{12} \partial_{t}^{4}+\ldots,
$$

we can represent (4) as an infinite series in $\epsilon$,

$$
u_{t t}-f(u)_{x x}-g(u)_{y y}+\frac{\epsilon^{2}}{12}\left[u_{t t t t}-f(u)_{x x x x}-g(u)_{y y y y}\right]+\cdots=0 .
$$

The corresponding dispersionless limit $\epsilon \rightarrow 0$ results in the quasilinear wave-type equation

$$
u_{t t}-f(u)_{x x}-g(u)_{y y}=0 .
$$

This equation possesses exact solutions of the form $u=R(x, y, t)$ where $R$ solves a pair of Hopf-type equations,

$$
R_{t}=\lambda(R) R_{x}, \quad R_{y}=\mu(R) R_{x},
$$

with the characteristic speeds $\lambda, \mu$ satisfying the dispersion relation $\lambda^{2}=f^{\prime}+g^{\prime} \mu^{2}$. Solutions of this type are known as one-phase hydrodynamic reductions, or planar simple waves. Let us require that all such reductions can be deformed into formal solutions of the original equation (4) as follows:

$$
\begin{aligned}
& R_{y}=\mu(R) R_{x}+\epsilon(\ldots)+\epsilon^{2}(\ldots)+\ldots, \\
& R_{t}=\lambda(R) R_{x}+\epsilon(\ldots)+\epsilon^{2}(\ldots)+\ldots,
\end{aligned}
$$

here dots at $\epsilon^{k}$ denote terms which are polynomial in the $x$-derivatives of $R$ of the order $k+1$. The relation $u=R(x, y, t)$ remains undeformed, this can always be assumed modulo the Miura group. We emphasise that such deformations are required to exist for any function $\mu(R)$. A direct calculation demonstrates that all terms of the order $\epsilon$ vanish identically, while at the order $\epsilon^{2}$ we get the following constraints for $f$ and $g$ :

$$
f^{\prime \prime}+g^{\prime \prime}=0, \quad g^{\prime \prime}\left(1+f^{\prime}\right)-g^{\prime} f^{\prime \prime}=0, \quad f^{\prime \prime 2}\left(1+2 f^{\prime}\right)-f^{\prime}\left(f^{\prime}+1\right) f^{\prime \prime \prime}=0 .
$$


Without any loss of generality one can set $f(u)=u-\ln \left(e^{u}+1\right), g(u)=\ln \left(e^{u}+1\right)$, resulting in the difference equation

$$
\triangle_{t \bar{t}} u-\triangle_{x \bar{x}}\left[u-\ln \left(e^{u}+1\right)\right]-\triangle_{y \bar{y}}\left[\ln \left(e^{u}+1\right)\right]=0,
$$

which is yet another equivalent form of the Hirota equation, known as the 'gauge-invariant form' [48], or the 'Y-system', see Appendix (we refer to [29] for a review of its applications). Its dispersionless limit,

$$
u_{t t}-\left[u-\ln \left(e^{u}+1\right)\right]_{x x}-\left[\ln \left(e^{u}+1\right)\right]_{y y}=0,
$$

appeared recently in the classification of integrable equations possessing the "central quadric ansatz' [19]. Note that the necessary conditions for integrability obtained at the order $\epsilon^{2}$ prove to be sufficient, that is, integrability 'to the order $\epsilon^{2}$, implies the integrability in conventional sense. This appears to be a general phenomenon, at least for all classification results of this paper. In the case (7), expansions (6) take the explicit form

$$
\begin{aligned}
& R_{y}=\mu(R) R_{x}+\epsilon^{2}\left(a_{1} R_{x x x}+a_{2} R_{x x} R_{x}+a_{3} R_{x}^{3}\right)+O\left(\epsilon^{4}\right), \\
& R_{t}=\lambda(R) R_{x}+\epsilon^{2}\left(b_{1} R_{x x x}+b_{2} R_{x x} R_{x}+b_{3} R_{x}^{3}\right)+O\left(\epsilon^{4}\right),
\end{aligned}
$$

where

$$
\begin{aligned}
& a_{1}=\frac{1}{12}\left(\mu^{2}-1\right) \mu^{\prime}, \\
& b_{1}=\frac{\left(\mu^{2}-1\right) e^{R}\left(\mu^{2}+2 \mu \mu^{\prime} e^{R}+2 \mu \mu^{\prime}-1\right)}{24\left(e^{R}+1\right)^{2} \lambda},
\end{aligned}
$$

etc. The remaining coefficients $a_{i}, b_{i}$ have a far more complicated structure, however, all of them are rational expressions in $\mu$ and its derivatives. Note that higher powers of $\lambda$ can be eliminated via the dispersion relation $\lambda^{2}=\frac{1}{e^{R}+1}+\frac{e^{R}}{e^{R}+1} \mu^{2}$.

We emphasise that our approach to the integrability in 3D is essentially intrinsic: it applies directly to a given equation, and does not require its embedding into a compatible hierarchy living in a higher dimensional space.

\section{$2.1 \quad$ Non-degeneracy conditions}

We have already mentioned that the method of dispersive deformations applies to 3D equations with a non-degenerate dispersionless limit. In general, this means that:

- the principal symbol of the dispersionless equation defines an irreducible algebraic curve, and

- the dispersionless equation is not linearly degenerate.

To be more specific, let us restrict to quasilinear PDEs of the form

$$
\sum_{i, j=1}^{3} f_{i j}\left(u_{k}\right) u_{i j}=0,
$$

that arise as dispersionless limits for most of the examples discussed in this paper; here the coefficients $f_{i j}$ depend on first-order derivatives $u_{k}$ only. In this case the first non-degeneracy 
condition is equivalent to $\operatorname{det} f_{i j} \neq 0$ (it is required for the applicability of the method of hydrodynamic reductions). To define the second non-degeneracy condition let us introduce the concept of linearly degenerate equations. These are characterised by the identity

$$
\partial_{(k} f_{i j)}=\varphi_{(k} f_{i j)},
$$

where $\partial_{k}=\partial_{u_{k}}, \varphi=\left(\varphi_{1}, \varphi_{2}, \varphi_{3}\right)$ is a covector, and brackets denote complete symmetrisation in $i, j, k \in\{1,2,3\}$. Explicitly, this gives ten relations:

$$
\begin{array}{cl}
\partial_{1} f_{11}=\varphi_{1} f_{11}, \quad \partial_{2} f_{22}=\varphi_{2} f_{22}, & \partial_{3} f_{33}=\varphi_{3} f_{33}, \\
\partial_{2} f_{11}+2 \partial_{1} f_{12}=\varphi_{2} f_{11}+2 \varphi_{1} f_{12}, & \partial_{1} f_{22}+2 \partial_{2} f_{12}=\varphi_{1} f_{22}+2 \varphi_{2} f_{12}, \\
\partial_{3} f_{11}+2 \partial_{1} f_{13}=\varphi_{3} f_{11}+2 \varphi_{1} f_{13}, & \partial_{1} f_{33}+2 \partial_{3} f_{13}=\varphi_{1} f_{33}+2 \varphi_{3} f_{13}, \\
\partial_{2} f_{33}+2 \partial_{3} f_{23}=\varphi_{2} f_{33}+2 \varphi_{3} f_{23}, & \partial_{3} f_{22}+2 \partial_{2} f_{23}=\varphi_{3} f_{22}+2 \varphi_{2} f_{23}, \\
\partial_{1} f_{23}+\partial_{2} f_{13}+\partial_{3} f_{12}=\varphi_{1} f_{23}+\varphi_{2} f_{13}+\varphi_{3} f_{12} .
\end{array}
$$

On elimination of $\varphi$ 's, these conditions give rise to seven first-order differential constraints for $f_{i j}$ alone. Linearly degenerate PDEs are quite exceptional from the point of view of solvability of the Cauchy problem: for these PDEs the gradient catastrophe, typical for genuinely nonlinear equations, does not occur, which implies global existence results for an open set of initial data. The reason for this is that linear degeneracy is closely related to the null conditions of Klainerman known in the theory of second-order quasilinear PDEs; we refer to [17] for further discussion and references.

It turns out that the method of dispersive deformations does not work for linearly degenerate PDEs: the conditions of linear degeneracy appear as denominators in the computation of dispersive corrections (to be precise, the denominator is a polynomial whose coefficients are conditions of linear degeneracy; it vanishes identically if and only if the equation is linearly degenerate). This phenomenon has a 'philosophical' explanation: dispersive terms are needed to prevent breakdown of smooth initial data; on the other hand, for linearly degenerate PDEs breakdown does not occur, in some sense linearly degenerate PDEs should be considered as 'dispersive', even without higher-order terms.

We point out that both non-degeneracy conditions are satisfied (possibly, after a change of variables) for all known examples of integrable PDEs in 3D.

\section{Discrete conservation laws in $3 \mathrm{D}$}

In this section we classify integrable equations of the form (3),

$$
\triangle_{1} f+\triangle_{2} g+\triangle_{3} h=0,
$$

where $f, g, h$ are functions of $\triangle_{1} u, \triangle_{2} u, \triangle_{3} u$ only. The corresponding dispersionless limit,

$$
\sum_{i, j=1}^{3} f_{i j}\left(u_{k}\right) u_{i j}=0,
$$

is assumed to be non-degenerate. The classification is performed modulo transformations of the form $u \rightarrow \alpha u+\alpha_{i} x^{i}$, as well as relabelling of the independent variables $x^{i}$. 
Theorem 1 Integrable discrete conservation laws are naturally grouped into seven three-parameter families,

$$
\alpha I+\beta J+\gamma K=0,
$$

where $\alpha, \beta, \gamma$ are arbitrary constants, while $I, J, K$ denote left hand sides of three linearly independent discrete conservation laws of the seven octahedron-type equations listed below. In each case we give explicit forms of $I, J, K$, as well as the underlying octahedron equation.

Case 1.

\begin{tabular}{|l|l|}
\hline Conservation Laws & Octahedron equation \\
\hline$I=\triangle_{1} e^{\triangle_{2} u}+\triangle_{3}\left(e^{\triangle_{2} u-\triangle_{1} u}-e^{\triangle_{2} u}\right)=0$ & $\frac{T_{2} \tau-T_{12} \tau}{T_{23} \tau}=T_{1} \tau\left(\frac{1}{T_{13} \tau}-\frac{1}{T_{3} \tau}\right)$ \\
$J=\triangle_{1} e^{-\triangle_{3} u}+\triangle_{2}\left(e^{\triangle_{1} u-\triangle_{3} u}-e^{-\triangle_{3} u}\right)=0$ & $\left(\right.$ setting $\left.\tau=e^{u / \epsilon}\right)$ \\
$K=\triangle_{2}\left(\triangle_{3} u-\ln \left(1-e^{\triangle_{1} u}\right)\right)+\triangle_{3}\left(\ln \left(1-e^{\triangle_{1} u}\right)-\triangle_{1} u\right)=0$ & \\
\hline
\end{tabular}

\section{Case 2.}

\begin{tabular}{|l|l|}
\hline Conservation Laws & Octahedron equation \\
\hline$I=\triangle_{2} \ln \triangle_{1} u+\triangle_{3} \ln \left(1-\frac{\triangle_{2} u}{\triangle_{1} u}\right)=0$ & $T_{12} u T_{13} u+T_{2} u T_{23} u+T_{1} u T_{3} u$ \\
$J=\triangle_{1} \ln \triangle_{2} u+\triangle_{3} \ln \left(\frac{\triangle_{1} u}{\triangle_{2} u}-1\right)=0$ & $=T_{12} u T_{23} u+T_{1} u T_{13} u+T_{2} u T_{3} u$ \\
$K=\triangle_{1}\left(\frac{\left(\triangle_{2} u\right)^{2}}{2}-\triangle_{2} u \triangle_{3} u\right)+\triangle_{2}\left(\triangle_{1} u \triangle_{3} u-\frac{\left(\triangle_{1} u\right)^{2}}{2}\right)=0$ & \\
\hline
\end{tabular}

Case 3. Generalised lattice Toda (depending on a parameter $\alpha$ )

\begin{tabular}{|c|c|}
\hline Conservation Laws & Octahedron equation \\
\hline $\begin{array}{l}\text { subcase } \alpha \neq 0 \\
I=\triangle_{1}\left(e^{\triangle_{2} u-\triangle_{3} u}+\alpha e^{-\triangle_{3} u}\right)-\triangle_{2}\left(e^{\triangle_{1} u-\triangle_{3} u}+\alpha e^{-\triangle_{3} u}\right)=0 \\
J=\triangle_{2} \ln \left(e^{\triangle_{1} u}+\alpha\right)+\triangle_{3}\left(\ln \frac{e^{\triangle_{1} u}-e^{\triangle_{2} u}}{e^{\triangle_{1} u}+\alpha}-\triangle_{2} u\right)=0 \\
K=\triangle_{1} \ln \left(e^{\triangle_{2} u}+\alpha\right)+\triangle_{3}\left(\ln \frac{e^{\triangle_{1} u}-e^{\triangle_{2} u}}{e^{\triangle_{2} u}+\alpha}-\triangle_{1} u\right)=0\end{array}$ & $\begin{array}{l}\frac{T_{23} \tau}{T_{3} \tau}+\frac{T_{12} \tau}{T_{2} \tau}+\alpha \frac{T_{12} \tau T_{23} \tau}{T_{2} \tau T_{3} \tau}= \\
\frac{T_{12} \tau}{T_{1} \tau}+\frac{T_{13} \tau}{T_{3} \tau}+\alpha \frac{T_{12} \tau T_{13} \tau}{T_{1} \tau T_{3} \tau} \\
\left(\text { setting } \tau=e^{-u / \epsilon}\right)\end{array}$ \\
\hline $\begin{array}{l}\text { subcase } \alpha=0 \\
I=\triangle_{1} e^{\triangle_{2} u-\triangle_{3} u}-\triangle_{2} e^{\triangle_{1} u-\triangle_{3} u}=0 \\
J=\triangle_{2} \triangle_{1} u+\triangle_{3}\left(\ln \left(1-e^{\triangle_{2} u-\triangle_{1} u}\right)-\triangle_{2} u\right)=0 \\
K=\triangle_{1} e^{-\triangle_{2} u}-\triangle_{2} e^{-\triangle_{1} u}+\triangle_{3}\left(e^{-\triangle_{1} u}-e^{-\triangle_{2} u}\right)=0\end{array}$ & $\begin{array}{l}\text { lattice Toda equation } \\
\left(T_{1}-T_{3}\right) \frac{T_{2} \tau}{\tau}=\left(T_{2}-T_{3}\right) \frac{T_{1} \tau}{\tau} \\
\left(\text { setting } \tau=e^{-u / \epsilon}\right)\end{array}$ \\
\hline
\end{tabular}

\section{Case 4. Lattice KP}

\begin{tabular}{|l|l|}
\hline Conservation Laws & Octahedron equation \\
\hline$I=\triangle_{1}\left(\left(\triangle_{3} u\right)^{2}-\left(\triangle_{2} u\right)^{2}\right)+\triangle_{2}\left(\left(\triangle_{1} u\right)^{2}\right.$ & $\left(T_{1} u-T_{2} u\right) T_{12} u+\left(T_{3} u-T_{1} u\right) T_{13} u$ \\
$\left.\quad-\left(\triangle_{3} u\right)^{2}\right)+\triangle_{3}\left(\left(\triangle_{2} u\right)^{2}-\left(\triangle_{1} u\right)^{2}\right)=0$ & $+\left(T_{2} u-T_{3} u\right) T_{23} u=0$ \\
$J=\triangle_{1} \ln \left(\triangle_{3} u-\triangle_{2} u\right)-\triangle_{2} \ln \left(\triangle_{1} u-\triangle_{3} u\right)=0$ & \\
$K=\triangle_{2} \ln \left(\triangle_{1} u-\triangle_{3} u\right)-\triangle_{3} \ln \left(\triangle_{2} u-\triangle_{1} u\right)=0$ & \\
\hline
\end{tabular}

\section{Case 5. Lattice mKP}

\begin{tabular}{|l|l|}
\hline Conservation Laws & Octahedron equation \\
\hline$I=\triangle_{1}\left(e^{\triangle_{2} u}-e^{\triangle_{3} u}\right)+\triangle_{2}\left(e^{\triangle_{3} u}-e^{\triangle_{1} u}\right)+\triangle_{3}\left(e^{\triangle_{1} u}-e^{\triangle_{2} u}\right)=0$ & $\frac{T_{13} \tau-T_{12} \tau}{T_{1} \tau}+\frac{T_{12} \tau-T_{23} \tau}{T_{2} \tau}$ \\
$J=\triangle_{1} \ln \left(e^{\triangle_{3} u}-e^{\triangle_{2} u}\right)-\triangle_{2} \ln \left(e^{\triangle_{3} u}-e^{\triangle_{1} u}\right)=0$ & $+\frac{T_{23} \tau-T_{13} \tau}{T_{3} \tau}=0$ \\
$K=\triangle_{2} \ln \left(e^{\triangle_{3} u}-e^{\triangle_{1} u}\right)-\triangle_{3} \ln \left(e^{\triangle_{2} u}-e^{\triangle_{1} u}\right)=0$ & (setting $\left.\tau=e^{u / \epsilon}\right)$ \\
\hline
\end{tabular}




\section{Case 6. Schwarzian KP}

\begin{tabular}{|l|l|}
\hline Conservation Laws & Octahedron equation \\
\hline$I=\triangle_{2} \ln \left(1-\frac{\triangle_{3} u}{\triangle_{1} u}\right)-\triangle_{3} \ln \left(\frac{\triangle_{2} u}{\triangle_{1} u}-1\right)=0$ & $\left(T_{2} \triangle_{1} u\right)\left(T_{3} \triangle_{2} u\right)\left(T_{1} \triangle_{3} u\right)$ \\
$J=\triangle_{3} \ln \left(1-\frac{\triangle_{1} u}{\triangle_{2} u}\right)-\triangle_{1} \ln \left(\frac{\triangle_{3} u}{\triangle_{2} u}-1\right)=0$ & $=\left(T_{2} \triangle_{3} u\right)\left(T_{3} \triangle_{1} u\right)\left(T_{1} \triangle_{2} u\right)$ \\
$K=\triangle_{1} \ln \left(1-\frac{\triangle_{2} u}{\triangle_{3} u}\right)-\triangle_{2} \ln \left(\frac{\triangle_{1} u}{\triangle_{3} u}-1\right)=0$ & \\
\hline
\end{tabular}

\section{Case 7. Lattice spin}

\begin{tabular}{|l|l|}
\hline Conservation Laws & Octahedron equation \\
\hline Hyperbolic version & lattice-spin equation \\
$I=\triangle_{1} \ln \frac{\sinh \triangle_{3} u}{\sinh \triangle_{2} u}+\triangle_{2} \ln \frac{\sinh \triangle_{1} u}{\sinh \triangle_{3} u}+\triangle_{3} \ln \frac{\sinh \triangle_{2} u}{\sinh \triangle_{1} u}=0$ & $\left(\frac{T_{12} \tau}{T_{2} \tau}-1\right)\left(\frac{T_{13} \tau}{T_{1} \tau}-1\right)\left(\frac{T_{23} \tau}{T_{3} \tau}-1\right)$ \\
$J=\triangle_{1} \ln \frac{\sinh \left(\triangle_{2} u-\triangle_{3} u\right)}{\sinh \triangle_{2} u}-\triangle_{3} \ln \frac{\sinh \left(\triangle_{1} u-\triangle_{2} u\right)}{\sinh \triangle_{2} u}=0$ & $=\left(\frac{T_{12} \tau}{T_{1} \tau}-1\right)\left(\frac{T_{13} \tau}{T_{3} \tau}-1\right)\left(\frac{T_{23} \tau}{T_{2} \tau}-1\right)$ \\
$K=\triangle_{2} \ln \frac{\sinh \left(\triangle_{3} u-\triangle_{1} u\right)}{\sinh \triangle_{1} u}-\triangle_{3} \ln \frac{\sinh \left(\triangle_{1} u-\triangle_{2} u\right)}{\sinh \triangle_{1} u}=0$ & $\left(\right.$ setting $\left.\tau=e^{2 u / \epsilon}\right)$ \\
\hline Trigonometric version & Sine-Gordon equation \\
$I=\triangle_{1} \ln \frac{\sin \triangle_{3} u}{\sin \triangle_{2} u}+\triangle_{2} \ln \frac{\sin \triangle_{1} u}{\sin \triangle_{3} u}+\triangle_{3} \ln \frac{\sin \triangle_{2} u}{\sin \triangle_{1} u}=0$ & $\left(T_{2} \sin \triangle_{1} u\right)\left(T_{3} \sin \triangle_{2} u\right)\left(T_{1} \sin \triangle_{3} u\right)$ \\
$J=\triangle_{1} \ln \frac{\left.\sin \triangle_{2} u-\triangle_{3} u\right)}{\sin \triangle_{2} u}-\triangle_{3} \ln \frac{\sin \left(\triangle_{1} u-\triangle_{2} u\right)}{\sin \triangle_{2} u}=0$ & $=\left(T_{2} \sin \triangle_{3} u\right)\left(T_{3} \sin \triangle_{1} u\right)\left(T_{1} \sin \triangle_{2} u\right)$ \\
$K=\triangle_{2} \ln \frac{\sin \left(\triangle_{3} u-\triangle_{1} u\right)}{\sin \triangle_{1} u}-\triangle_{3} \ln \frac{\sin \left(\triangle_{1} u-\triangle_{2} u\right)}{\sin \triangle_{1} u}=0$ & \\
\hline
\end{tabular}

Remark. Although the cases 1, 2 do not bear any special name, the corresponding equations can be obtained as degenerations from 3-7. Furthermore, they are contained in the classification of [3].

\section{Proof of Theorem 1:}

The dispersionless limit of (3) is a quasilinear conservation law

$$
\partial_{1} f+\partial_{2} g+\partial_{3} h=0
$$

where $f, g, h$ are functions of the variables $a=u_{1}, b=u_{2}, c=u_{3}$. Requiring that all one-phase reductions of the dispersionless equation (9) are inherited by the discrete equation (3) we obtain a set of differential constraints for $f, g, h$, that are the necessary conditions for integrability. Thus, at the order $\epsilon$ we get

$$
f_{a}=g_{b}=h_{c}=0, \quad f_{b}+g_{a}+f_{c}+h_{a}+g_{c}+h_{b}=0 .
$$

The first set of these relations implies that the dispersionless limit is equivalent to the secondorder PDE

$$
F u_{12}+G u_{13}+H u_{23}=0,
$$

where $F=f_{b}+g_{a}, G=f_{c}+h_{a}, H=g_{c}+h_{b}$. Note that, by virtue of (10), the coefficients $F, G, H$ satisfy the additional constraint $F+G+H=0$. It follows from [9] that, up to a non-zero factor, any integrable equation of this type is equivalent to

$$
\left[p\left(u_{1}\right)-q\left(u_{2}\right)\right] u_{12}+\left[r\left(u_{3}\right)-p\left(u_{1}\right)\right] u_{13}+\left[q\left(u_{2}\right)-r\left(u_{3}\right)\right] u_{23}=0,
$$


where the functions $p(a), q(b), r(c)$ satisfy the integrability conditions

$$
\begin{aligned}
& p^{\prime \prime}=p^{\prime}\left(\frac{p^{\prime}-q^{\prime}}{p-q}+\frac{p^{\prime}-r^{\prime}}{p-r}-\frac{q^{\prime}-r^{\prime}}{q-r}\right), \\
& q^{\prime \prime}=q^{\prime}\left(\frac{q^{\prime}-p^{\prime}}{q-p}+\frac{q^{\prime}-r^{\prime}}{q-r}-\frac{p^{\prime}-r^{\prime}}{p-r}\right), \\
& r^{\prime \prime}=r^{\prime}\left(\frac{r^{\prime}-p^{\prime}}{r-p}+\frac{r^{\prime}-q^{\prime}}{r-q}-\frac{p^{\prime}-q^{\prime}}{p-q}\right) .
\end{aligned}
$$

Our further strategy can be summarised as follows:

Step 1. First, we solve equations (13). Modulo unessential translations and rescalings this leads to seven quasilinear integrable equations of the form (12), see the details below.

Step 2. Next, for all of the seven equations found at step 1, we calculate first-order conservation laws. It was demonstrated in [9] that any integrable second-order quasilinear PDE possesses exactly four conservation laws of the form (9).

Step 3. Taking linear combinations of the four conservation laws in each of the above seven cases, and replacing partial derivatives $u_{1}, u_{2}, u_{3}$ by discrete derivatives $\triangle_{1} u, \triangle_{2} u, \triangle_{3} u$, we obtain discrete equations (3) which, at this stage, are the candidates for integrability.

Step 4. Applying the $\epsilon^{2}$-integrability test, we obtain constraints for the coefficients of linear combinations. It turns out that only linear combinations of three (out of four) conservation laws pass the integrability test. In what follows, we present conservation laws in such a way that the first three are the ones that pass the integrability test, while the fourth one doesn't. Each triplet of conservation laws corresponds to one and the same discrete integrable equation of octahedron type. In other words, there are overall seven discrete integrable equations of octahedron type, each of them possesses three conservation laws, and linear combinations thereof give all integrable examples of the form (3).

Let us proceed to the solution of the system (13). There are three essentially different cases to consider, depending on how many functions among $p, q, r$ are constant (the case when all of them are constant corresponds to linear equations). Some of these cases have additional subcases. These correspond to the seven cases of Theorem 1, in the same order as they appear below (note that the labelling below is different, dictated by the logic of the classification procedure).

Case 1: $q$ and $r$ are distinct constants. Without any loss of generality one can set $q=1, r=-1$. In this case the equations for $q$ and $r$ will be satisfied identically, while the equation for $p$ takes the form $p^{\prime \prime}=2 p p^{2} /\left(p^{2}-1\right)$. Modulo unessential scaling parameters this gives $p=\left(1+e^{u_{1}}\right) /\left(1-e^{u_{1}}\right)$, resulting in the $\mathrm{PDE}$

$$
e^{u_{1}} u_{12}-u_{13}+\left(1-e^{u_{1}}\right) u_{23}=0
$$

This equation possesses four conservation laws:

$$
\begin{gathered}
\partial_{1} e^{u_{2}}+\partial_{3}\left(e^{u_{2}-u_{1}}-e^{u_{2}}\right)=0, \\
\partial_{1} e^{-u_{3}}+\partial_{2}\left(e^{u_{1}-u_{3}}-e^{-u_{3}}\right)=0, \\
\partial_{2}\left(u_{3}-\ln \left(1-e^{u_{1}}\right)\right)+\partial_{3}\left(\ln \left(1-e^{u_{1}}\right)-u_{1}\right)=0,
\end{gathered}
$$


$\partial_{1}\left(\frac{u_{2} u_{3}}{2}\right)-\partial_{2}\left(\frac{u_{1} u_{3}}{2}-u_{1} \ln \left(1-e^{u_{1}}\right)-L i_{2}\left(e^{u_{1}}\right)\right)+\partial_{3}\left(\frac{u_{1}^{2}}{2}-\frac{u_{1} u_{2}}{2}-u_{1} \ln \left(1-e^{u_{1}}\right)-L i_{2}\left(e^{u_{1}}\right)\right)=0$

where $L i_{2}$ is the dilogarithm function, $L i_{2}(z)=-\int \frac{\ln (1-z)}{z} d z$. Applying steps 3 and 4 , one can show that discrete versions of the first three conservation laws correspond to the discrete equation

$$
e^{\left(T_{1} u-T_{13} u\right) / \epsilon}+e^{\left(T_{12} u-T_{23} u\right) / \epsilon}=e^{\left(T_{1} u-T_{3} u\right) / \epsilon}+e^{\left(T_{2} u-T_{23} u\right) / \epsilon} .
$$

Setting $\tau=e^{u / \epsilon}$ it can be rewritten as

$$
\frac{T_{2} \tau-T_{12} \tau}{T_{23} \tau}=T_{1} \tau\left(\frac{1}{T_{13} \tau}-\frac{1}{T_{3} \tau}\right)
$$

Case 2: $r$ is constant. Without any loss of generality one can set $r=0$. In this case the above system of ODEs for $p$ and $q$ takes the form

$$
\frac{p^{\prime \prime}}{p^{\prime}}=\frac{p^{\prime}-q^{\prime}}{p-q}+\frac{p^{\prime}}{p}-\frac{q^{\prime}}{q}, \quad \frac{q^{\prime \prime}}{q^{\prime}}=\frac{p^{\prime}-q^{\prime}}{p-q}+\frac{q^{\prime}}{q}-\frac{p^{\prime}}{p} .
$$

Subtraction of these equations and the separation of variables leads, modulo unessential rescalings, to the two different subcases.

subcase 2a: $p=1 / u_{1}, q=1 / u_{2}$. The corresponding PDE is

$$
\left(u_{2}-u_{1}\right) u_{12}-u_{2} u_{13}+u_{1} u_{23}=0 .
$$

It possesses four conservation laws:

$$
\begin{gathered}
\partial_{2} \ln u_{1}+\partial_{3} \ln \left(1-\frac{u_{2}}{u_{1}}\right)=0, \\
\partial_{1} \ln u_{2}+\partial_{3} \ln \left(\frac{u_{1}}{u_{2}}-1\right)=0, \\
\partial_{1}\left(u_{2}^{2}-2 u_{2} u_{3}\right)+\partial_{2}\left(2 u_{1} u_{3}-u_{1}^{2}\right)=0, \\
\partial_{1}\left(-\frac{2 u_{2}^{3}}{9}+u_{2}^{2} u_{3}-u_{2} u_{3}^{2}\right)+\partial_{2}\left(\frac{2 u_{1}^{3}}{9}-u_{1}^{2} u_{3}+u_{1} u_{3}^{2}\right)+\partial_{3}\left(\frac{u_{1}^{2} u_{2}-u_{1} u_{2}^{2}}{3}\right)=0 .
\end{gathered}
$$

Applying steps 3 and 4, one can show that discrete versions of the first three conservation laws correspond to the discrete equation

$$
T_{12} u T_{13} u+T_{2} u T_{23} u+T_{1} u T_{3} u=T_{12} u T_{23} u+T_{1} u T_{13} u+T_{2} u T_{3} u .
$$

subcase 2b: $p=1 /\left(e^{u_{1}}+\alpha\right), q=1 /\left(e^{u_{2}}+\alpha\right), \alpha=$ const. The corresponding PDE is

$$
\left(e^{u_{2}}-e^{u_{1}}\right) u_{12}-\left(e^{u_{2}}+\alpha\right) u_{13}+\left(e^{u_{1}}+\alpha\right) u_{23}=0 .
$$

If $\alpha \neq 0$ it possesses the following four conservation laws:

$$
\begin{gathered}
\partial_{1}\left(e^{u_{2}-u_{3}}+\alpha e^{-u_{3}}\right)-\partial_{2}\left(e^{u_{1}-u_{3}}+\alpha e^{-u_{3}}\right)=0 \\
\partial_{2} \ln \left(e^{u_{1}}+\alpha\right)+\partial_{3}\left(\ln \frac{e^{u_{1}}-e^{u_{2}}}{e^{u_{1}}+\alpha}-u_{2}\right)=0
\end{gathered}
$$




$$
\begin{gathered}
\partial_{1} \ln \left(e^{u_{2}}+\alpha\right)+\partial_{3}\left(\ln \frac{e^{u_{1}}-e^{u_{2}}}{e^{u_{2}}+\alpha}-u_{1}\right)=0, \\
\partial_{1}\left(-u_{2} u_{3}+2 u_{2} \ln \left(\frac{e^{u_{2}}+\alpha}{\alpha}\right)+2 L i_{2}\left(-\frac{e^{u_{2}}}{\alpha}\right)\right)+\partial_{2}\left(u_{1} u_{3}-2 u_{1} \ln \left(\frac{e^{u_{1}}+\alpha}{\alpha}\right)-2 L i_{2}\left(-\frac{e^{u_{1}}}{\alpha}\right)\right) \\
+\partial_{3}\left(u_{2}^{2}-u_{1} u_{2}+2\left(u_{2}-u_{1}\right) \ln \left(1-e^{u_{1}-u_{2}}\right)+2 u_{1} \ln \left(\frac{e^{u_{1}}+\alpha}{\alpha}\right)-2 u_{2} \ln \left(\frac{e^{u_{2}}+\alpha}{\alpha}\right)\right. \\
\left.+2 L i_{2}\left(-\frac{e^{u_{1}}}{\alpha}\right)-2 L i_{2}\left(-\frac{e^{u_{2}}}{\alpha}\right)-2 L i_{2}\left(e^{u_{1}-u_{2}}\right)\right)=0,
\end{gathered}
$$

while when $\alpha=0$ the conservation laws take the form:

$$
\begin{gathered}
\partial_{1} e^{u_{2}-u_{3}}-\partial_{2} e^{u_{1}-u_{3}}=0, \\
\partial_{2} u_{1}+\partial_{3}\left(\ln \left(1-e^{u_{2}-u_{1}}\right)-u_{2}\right)=0, \\
\partial_{1} e^{-u_{2}}-\partial_{2} e^{-u_{1}}+\partial_{3}\left(e^{-u_{1}}-e^{-u_{2}}\right)=0, \\
\partial_{1}\left(u_{2}^{2}-u_{2} u_{3}\right)+\partial_{2}\left(u_{1} u_{3}-u_{1}^{2}\right)+\partial_{3}\left(u_{1}^{2}-u_{1} u_{2}+2\left(u_{2}-u_{1}\right) \ln \left(1-e^{u_{1}-u_{2}}\right)-2 L i_{2}\left(e^{u_{1}-u_{2}}\right)\right)=0 .
\end{gathered}
$$

Applying steps 3 and 4 to the subcase $\alpha \neq 0$, one can show that discrete versions of the first three conservation laws correspond to the discrete equation

$$
\begin{gathered}
e^{\left(T_{3} u-T_{23} u\right) / \epsilon}+e^{\left(T_{2} u-T_{12} u\right) / \epsilon}+\alpha e^{\left(T_{2} u+T_{3} u-T_{12} u-T_{23} u\right) / \epsilon}= \\
e^{\left(T_{3} u-T_{13} u\right) / \epsilon}+e^{\left(T_{1} u-T_{12} u\right) / \epsilon}+\alpha e^{\left(T_{1} u+T_{3} u-T_{12} u-T_{13} u\right.} .
\end{gathered}
$$

Setting $\tau=e^{-u / \epsilon}$, this equation can be rewritten as

$$
\frac{T_{23} \tau}{T_{3} \tau}+\frac{T_{12} \tau}{T_{2} \tau}+\alpha \frac{T_{12} \tau T_{23} \tau}{T_{2} \tau T_{3} \tau}=\frac{T_{12} \tau}{T_{1} \tau}+\frac{T_{13} \tau}{T_{3} \tau}+\alpha \frac{T_{12} \tau T_{13} \tau}{T_{1} \tau T_{3} \tau}
$$

The special case $\alpha=0$ leads to the lattice Toda equation,

$$
\left(T_{1}-T_{3}\right) \frac{T_{2} \tau}{\tau}=\left(T_{2}-T_{3}\right) \frac{T_{1} \tau}{\tau},
$$

see Appendix.

Case 3: none of $p, q, r$ are constant. In this case we can separate the variables in (13) as follows. Dividing equations (13) by $p^{\prime}, q^{\prime}, r^{\prime}$ respectively, and adding the first two of them we obtain

$$
p^{\prime \prime} / p^{\prime}+q^{\prime \prime} / q^{\prime}=2\left(p^{\prime}-q^{\prime}\right) /(p-q) .
$$

Multiplying both sides by $p-q$ and applying the operator $\partial_{a} \partial_{b}$ we obtain $\left(p^{\prime \prime} / p^{\prime}\right)^{\prime}=2 \alpha p^{\prime},\left(q^{\prime \prime} / q^{\prime}\right)^{\prime}=$ $2 \alpha q^{\prime}, \alpha=$ const. Thus, $p^{\prime \prime} / p^{\prime}=2 \alpha p+\beta_{1}, q^{\prime \prime} / q^{\prime}=2 \alpha q+\beta_{2}$. Substituting these expressions back into the above relation we obtain that $p^{\prime}$ and $q^{\prime}$ must be (the same) quadratic polynomials in $p$ and $q$, respectively. Ultimately,

$$
p^{\prime}=\alpha p^{2}+\beta p+\gamma, \quad q^{\prime}=\alpha q^{2}+\beta q+\gamma, \quad r^{\prime}=\alpha r^{2}+\beta r+\gamma .
$$

Modulo unessential translations and rescalings, this leads to the four subcases. 
subcase 3a: $p=u_{1}, q=u_{2}, r=u_{3}$. The corresponding PDE is

$$
\left(u_{2}-u_{1}\right) u_{12}+\left(u_{1}-u_{3}\right) u_{13}+\left(u_{3}-u_{2}\right) u_{23}=0 .
$$

It possesses four conservation laws:

$$
\begin{gathered}
\partial_{1}\left(u_{3}^{2}-u_{2}^{2}\right)+\partial_{2}\left(u_{1}^{2}-u_{3}^{2}\right)+\partial_{3}\left(u_{2}^{2}-u_{1}^{2}\right)=0, \\
\alpha_{1} \partial_{1} \ln \left(u_{3}-u_{2}\right)+\alpha_{2} \partial_{2} \ln \left(u_{1}-u_{3}\right)+\alpha_{3} \partial_{3} \ln \left(u_{2}-u_{1}\right)=0, \\
\partial_{1}\left(\frac{u_{3}^{3}-u_{2}^{3}}{3}+\frac{u_{2} u_{3}^{2}-u_{2}^{2} u_{3}}{2}\right)+\partial_{2}\left(\frac{u_{1}^{3}-u_{3}^{3}}{3}+\frac{u_{3} u_{1}^{2}-u_{3}^{2} u_{1}}{2}\right)+\partial_{3}\left(\frac{u_{2}^{3}-u_{1}^{3}}{3}+\frac{u_{1} u_{2}^{2}-u_{1}^{2} u_{2}}{2}\right)=0,
\end{gathered}
$$

where $\alpha_{1}, \alpha_{2}, \alpha_{3}$ are constants satisfying $\alpha_{1}+\alpha_{2}+\alpha_{3}=0$. Applying steps 3 and 4 , one can show that discrete versions of the first three conservation laws correspond to the discrete equation

$$
\left(T_{1} u-T_{2} u\right) T_{12} u+\left(T_{3} u-T_{1} u\right) T_{13} u+\left(T_{2} u-T_{3} u\right) T_{23} u=0
$$

which is known as the lattice KP equation (see Appendix).

subcase 3b: $p=e^{u_{1}}, q=e^{u_{2}}, r=e^{u_{3}}$. The corresponding PDE is

$$
\left(e^{u_{1}}-e^{u_{2}}\right) u_{12}+\left(e^{u_{3}}-e^{u_{1}}\right) u_{13}+\left(e^{u_{2}}-e^{u_{3}}\right) u_{23}=0 .
$$

It possesses four conservation laws:

$$
\begin{gathered}
\partial_{1}\left(e^{u_{2}}-e^{u_{3}}\right)+\partial_{2}\left(e^{u_{3}}-e^{u_{1}}\right)+\partial_{3}\left(e^{u_{1}}-e^{u_{2}}\right)=0, \\
\partial_{1} \ln \left(e^{u_{3}}-e^{u_{2}}\right)-\partial_{2} \ln \left(e^{u_{3}}-e^{u_{1}}\right)=0, \\
\partial_{2} \ln \left(e^{u_{3}}-e^{u_{1}}\right)-\partial_{3} \ln \left(e^{u_{2}}-e^{u_{1}}\right)=0, \\
\partial_{1}\left(u_{2} u_{3}-u_{3}^{2}+2\left(u_{2}-u_{3}-1\right) \ln \left(1-e^{u_{2}-u_{3}}\right)+2 L i_{2}\left(e^{u_{2}-u_{3}}\right)\right)+ \\
\partial_{2}\left(u_{3}^{2}-u_{1} u_{3}+2\left(u_{3}-u_{1}+1\right) \ln \left(1-e^{u_{1}-u_{3}}\right)-2 L i_{2}\left(e^{u_{1}-u_{3}}\right)\right)+ \\
\partial_{3}\left(u_{1} u_{2}-u_{2}^{2}-2\left(u_{1}-u_{2}\right)+2\left(u_{1}-u_{2}\right) \ln \left(1-e^{u_{1}-u_{2}}\right)+2 L i_{2}\left(e^{u_{1}-u_{2}}\right)\right)=0 .
\end{gathered}
$$

Again, applying steps 3 and 4, one can show that discrete versions of the first three conservation laws correspond to the discrete equation

$$
e^{-\frac{T_{1} u}{\epsilon}}\left(e^{\frac{T_{13} u}{\epsilon}}-e^{\frac{T_{12} u}{\epsilon}}\right)+e^{-\frac{T_{2} u}{\epsilon}}\left(e^{\frac{T_{12} u}{\epsilon}}-e^{\frac{T_{23} u}{\epsilon}}\right)+e^{-\frac{T_{3} u}{\epsilon}}\left(e^{\frac{T_{23} u}{\epsilon}}-e^{\frac{T_{13} u}{\epsilon}}\right)=0 .
$$

Setting $\tau=e^{u / \epsilon}$, this takes the form

$$
\frac{T_{13} \tau-T_{12} \tau}{T_{1} \tau}+\frac{T_{12} \tau-T_{23} \tau}{T_{2} \tau}+\frac{T_{23} \tau-T_{13} \tau}{T_{3} \tau}=0,
$$

which is known as the lattice mKP equation (see Appendix).

subcase 3c: $p=1 / u_{1}, q=1 / u_{2}, r=1 / u_{3}$. The corresponding PDE is

$$
u_{3}\left(u_{2}-u_{1}\right) u_{12}+u_{2}\left(u_{1}-u_{3}\right) u_{13}+u_{1}\left(u_{3}-u_{2}\right) u_{23}=0 .
$$


It possesses four conservation laws:

$$
\begin{gathered}
\partial_{2} \ln \left(1-\frac{u_{3}}{u_{1}}\right)-\partial_{3} \ln \left(\frac{u_{2}}{u_{1}}-1\right)=0, \\
\partial_{3} \ln \left(1-\frac{u_{1}}{u_{2}}\right)-\partial_{1} \ln \left(\frac{u_{3}}{u_{2}}-1\right)=0, \\
\partial_{1} \ln \left(1-\frac{u_{2}}{u_{3}}\right)-\partial_{2} \ln \left(\frac{u_{1}}{u_{3}}-1\right)=0, \\
\partial_{1}\left(u_{2}^{2} u_{3}-u_{2} u_{3}^{2}\right)+\partial_{2}\left(u_{3}^{2} u_{1}-u_{3} u_{1}^{2}\right)+\partial_{3}\left(u_{1}^{2} u_{2}-u_{1} u_{2}^{2}\right)=0 .
\end{gathered}
$$

Applying steps 3 and 4, one can show that discrete versions of the first three conservation laws correspond to the discrete equation

$$
\left(T_{2} \triangle_{1} u\right)\left(T_{3} \triangle_{2} u\right)\left(T_{1} \triangle_{3} u\right)=\left(T_{2} \triangle_{3} u\right)\left(T_{3} \triangle_{1} u\right)\left(T_{1} \triangle_{2} u\right),
$$

known as the Schwarzian KP equation (see Appendix).

subcase 3d: $p=\operatorname{coth} u_{1}, q=\operatorname{coth} u_{2}, r=\operatorname{coth} u_{3}$ (one can also take the trigonometric version coth $\rightarrow$ cot). The corresponding PDE is

$$
\left(\operatorname{coth} u_{2}-\operatorname{coth} u_{1}\right) u_{12}+\left(\operatorname{coth} u_{1}-\operatorname{coth} u_{3}\right) u_{13}+\left(\operatorname{coth} u_{3}-\operatorname{coth} u_{2}\right) u_{23}=0 .
$$

It possesses four conservation laws:

$$
\begin{gathered}
\partial_{1} \ln \frac{\sinh u_{3}}{\sinh u_{2}}+\partial_{2} \ln \frac{\sinh u_{1}}{\sinh u_{3}}+\partial_{3} \ln \frac{\sinh u_{2}}{\sinh u_{1}}=0, \\
\partial_{1} \ln \frac{\sinh \left(u_{2}-u_{3}\right)}{\sinh u_{2}}-\partial_{3} \ln \frac{\sinh \left(u_{1}-u_{2}\right)}{\sinh u_{2}}=0, \\
\partial_{2} \ln \frac{\sinh \left(u_{3}-u_{1}\right)}{\sinh u_{1}}-\partial_{3} \ln \frac{\sinh \left(u_{1}-u_{2}\right)}{\sinh u_{1}}=0, \\
\partial_{1}\left(-2 u_{3}^{2}+2 u_{2} u_{3}-2 u_{2} \ln \frac{\sinh \left(u_{2}-u_{3}\right)}{\sinh u_{2}}+\left(2 u_{3}-1\right) \ln \frac{\sinh \left(u_{2}-u_{3}\right)}{\sinh u_{3}}+\right. \\
\left.L i_{2}\left(e^{2 u_{2}}\right)-L i_{2}\left(e^{2 u_{3}}\right)-L i_{2}\left(e^{2\left(u_{2}-u_{3}\right)}\right)\right)+ \\
\partial_{2}\left(2 u_{3}^{2}-2 u_{1} u_{3}+\left(2 u_{1}-1\right) \ln \frac{\sinh \left(u_{3}-u_{1}\right)}{\sinh u_{1}}+\left(1-2 u_{3}\right) \ln \frac{\sinh \left(u_{3}-u_{1}\right)}{\sinh u_{3}}-\right. \\
\left.L i_{2}\left(e^{2 u_{1}}\right)+L i_{2}\left(e^{2 u_{3}}\right)+L i_{2}\left(e^{2\left(u_{1}-u_{3}\right)}\right)\right)+ \\
\partial_{3}\left(-2 u_{2}^{2}+2 u_{1} u_{2}+2 u_{2} \ln \frac{\sinh \left(u_{1}-u_{2}\right)}{\sinh u_{2}}+\left(1-2 u_{1}\right) \ln \frac{\sinh \left(u_{1}-u_{2}\right)}{\sinh u_{1}}+\right. \\
\left.L i_{2}\left(e^{2 u_{1}}\right)-L i_{2}\left(e^{2 u_{2}}\right)-L i_{2}\left(e^{2\left(u_{1}-u_{2}\right)}\right)\right)=0 .
\end{gathered}
$$

Applying steps 3 and 4, one can show that discrete versions of the first three conservation laws correspond to the discrete equation

$$
\begin{gathered}
\left(e^{2\left(T_{12} u-T_{2} u\right) / \epsilon}-1\right)\left(e^{2\left(T_{13} u-T_{1} u\right) / \epsilon}-1\right)\left(e^{2\left(T_{23} u-T_{3} u\right) / \epsilon}-1\right)= \\
\left(e^{2\left(T_{12} u-T_{1} u\right) / \epsilon}-1\right)\left(e^{2\left(T_{13} u-T_{3} u\right) / \epsilon}-1\right)\left(e^{2\left(T_{23} u-T_{2} u\right) / \epsilon}-1\right) .
\end{gathered}
$$


Setting $\tau=e^{2 u / \epsilon}$, it can be rewritten as

$$
\left(\frac{T_{12} \tau}{T_{2} \tau}-1\right)\left(\frac{T_{13} \tau}{T_{1} \tau}-1\right)\left(\frac{T_{23} \tau}{T_{3} \tau}-1\right)=\left(\frac{T_{12} \tau}{T_{1} \tau}-1\right)\left(\frac{T_{13} \tau}{T_{3} \tau}-1\right)\left(\frac{T_{23} \tau}{T_{2} \tau}-1\right)
$$

which is known as the lattice spin equation (see Appendix). In the trigonometric case, one can show that discrete versions of the conservation laws

$$
\begin{aligned}
& \partial_{1} \ln \frac{\sin u_{3}}{\sin u_{2}}+\partial_{2} \ln \frac{\sin u_{1}}{\sin u_{3}}+\partial_{3} \ln \frac{\sin u_{2}}{\sin u_{1}}=0, \\
& \partial_{1} \ln \frac{\sin \left(u_{2}-u_{3}\right)}{\sin u_{2}}-\partial_{3} \ln \frac{\sin \left(u_{1}-u_{2}\right)}{\sin u_{2}}=0, \\
& \partial_{2} \ln \frac{\sin \left(u_{3}-u_{1}\right)}{\sin u_{1}}-\partial_{3} \ln \frac{\sin \left(u_{1}-u_{2}\right)}{\sin u_{1}}=0,
\end{aligned}
$$

correspond to the discrete Sine-Gordon equation,

$$
\left(T_{2} \sin \triangle_{1} u\right)\left(T_{3} \sin \triangle_{2} u\right)\left(T_{1} \sin \triangle_{3} u\right)=\left(T_{2} \sin \triangle_{3} u\right)\left(T_{3} \sin \triangle_{1} u\right)\left(T_{1} \sin \triangle_{2} u\right) .
$$

This finishes the proof of Theorem 1.

Remark. It was observed in [32] that the Lagrangians $L\left(u, u_{1}, u_{2} ; \alpha_{1}, \alpha_{2}\right)$ of $2 \mathrm{D}$ discrete integrable equations of the ABS type [2] satisfy the closure relations

$$
\triangle_{1} L\left(u, u_{2}, u_{3} ; \alpha_{2}, \alpha_{3}\right)+\triangle_{2} L\left(u, u_{3}, u_{1} ; \alpha_{3}, \alpha_{1}\right)+\triangle_{3} L\left(u, u_{1}, u_{2} ; \alpha_{1}, \alpha_{2}\right)=0,
$$

which can be interpreted as 3D discrete conservation laws. For instance, the $Q_{1}$ case corresponds to the Lagrangian

$$
L\left(u, u_{1}, u_{2} ; \alpha_{1}, \alpha_{2}\right)=\alpha_{2} \ln \left(1-\frac{\triangle_{1} u}{\triangle_{2} u}\right)-\alpha_{1} \ln \left(\frac{\triangle_{2} u}{\triangle_{1} u}-1\right) .
$$

Remarkably, the corresponding closure relation (14), viewed as a single $3 \mathrm{D}$ equation, turns out to be integrable (subcase 6 of Theorem 1). Note that the constraint $\alpha_{1}=\alpha_{2}=\alpha_{3}$ reduces (14) to the Schwarzian KP equation,

$$
\triangle_{1}\left(\ln \frac{\triangle_{3} u}{\triangle_{2} u}\right)+\triangle_{2}\left(\ln \frac{\triangle_{1} u}{\triangle_{3} u}\right)+\triangle_{3}\left(\ln \frac{\triangle_{2} u}{\triangle_{1} u}\right)=0 .
$$

On the contrary, closure relations corresponding to the Lagrangians containing the dilogarithm $L i_{2}$ fail the $\epsilon^{2}$ integrability test. We refer to [4] for further connections between ABS equations and $3 \mathrm{D}$ integrable equations of octahedron type.

\subsection{Two discrete and one continuous variables.}

In this subsection we classify conservative equations of the form

$$
\triangle_{1} f+\triangle_{2} g+\partial_{3} h=0,
$$

where $f, g, h$ are functions of $\triangle_{1} u, \triangle_{2} u, u_{3}$. Again, non-degeneracy of the dispersionless limit is assumed. Our classification result is as follows: 
Theorem 2 Integrable equations of the form (15) are grouped into seven three-parameter families,

$$
\alpha I+\beta J+\gamma K=0,
$$

where $\alpha, \beta, \gamma$ are arbitrary constants, while $I, J, K$ denote left hand sides of three linearly independent semi-discrete conservation laws of the seven differential-difference equations listed below. In each case we give explicit forms of $I, J, K$, as well as the underlying differential-difference equation.

\section{Case 1.}

\begin{tabular}{|l|l|}
\hline Conservation Laws & Differential-difference equation \\
\hline$I=\triangle_{1} e^{\triangle_{2} u}-\partial_{3} e^{\triangle_{2} u-\triangle_{1} u}=0$ & \\
$J=\triangle_{1} u_{3}+\triangle_{2}\left(e^{\triangle_{1} u}-u_{3}\right)=0$ & $\frac{T_{12} v}{T_{2} v}+\frac{T_{1} v_{3}}{T_{1} v}=\frac{T_{1} v}{v}+\frac{T_{2} v_{3}}{T_{2} v}$ \\
$K=\triangle_{1} u_{3}^{2}+\triangle_{2}\left(2 e^{\triangle_{1} u} u_{3}-e^{2 \triangle_{1} u}-u_{3}^{2}\right)-\partial_{3}\left(2 e^{\triangle_{1} u}\right)=0$ & (setting $\left.v=e^{u / \epsilon}, \partial_{3} \rightarrow \frac{1}{\epsilon} \partial_{3}\right)$ \\
& \\
\hline
\end{tabular}

\section{Case 2.}

\begin{tabular}{|l|l|}
\hline Conservation Laws & Differential-difference equation \\
\hline $\begin{array}{l}I=\triangle_{1}\left(e^{\triangle_{2} u}-u_{3}\right)+\partial_{3} \ln \left(e^{\triangle_{1} u}-e^{\triangle_{2} u}\right)=0 \\
J=\triangle_{2}\left(e^{\triangle_{1} u}-u_{3}\right)+\partial_{3} \ln \left(e^{\triangle_{1} u}-e^{\triangle_{2} u}\right)=0\end{array}$ & $T_{12} v=\frac{T_{1} v T_{2} v}{v}+\frac{T_{2} v T_{1} v_{3}-T_{1} v T_{2} v_{3}}{T_{2} v-T_{1} v}$ \\
$K=\triangle_{1}\left(e^{2 \triangle_{2} u}-2 e^{\triangle_{2} u} u_{3}+u_{3}^{2}\right)+\triangle_{2}\left(2 e^{\triangle_{1} u} u_{3}-e^{2 \triangle_{1} u}-\right.$ & $\left(\right.$ setting $\left.v=e^{u / \epsilon}, \partial_{3} \rightarrow \frac{1}{\epsilon} \partial_{3}\right)$ \\
& \\
$\left.u_{3}^{2}\right)+\partial_{3}\left(2 e^{\triangle_{2} u}-2 e^{\triangle_{1} u}\right)=0$ & \\
\hline
\end{tabular}

\section{Case 3.}

\begin{tabular}{|l|l|}
\hline Conservation Laws & Differential-difference equation \\
\hline$I=\triangle_{1}\left(e^{\triangle_{2} u} u_{3}\right)-\partial_{3} e^{\triangle_{2} u}=0$ & $\frac{v T_{12} v}{T_{1} v}=\frac{T_{1} v T_{2} v_{3}}{T_{1} v_{3}} \quad\left(\right.$ setting $\left.v=e^{u / \epsilon}\right)$ \\
$J=\triangle_{2}\left(e^{-\triangle_{1} u} u_{3}\right)+\partial_{3} e^{-\triangle_{1} u}=0$ & \\
$K=\triangle_{1}\left(\triangle_{2} u+\ln u_{3}\right)-\triangle_{2} \ln u_{3}=0$ & \\
\hline
\end{tabular}

\section{Case 4.}

\begin{tabular}{|l|l|}
\hline Conservation Laws & Differential-difference equation \\
\hline$I=\triangle_{2}\left(\frac{u_{3}}{\triangle_{1} u}\right)-\partial_{3} \ln \left(\triangle_{1} u\right)=0$ & \\
$J=\triangle_{1} \ln u_{3}+\triangle_{2} \ln \left(\frac{\triangle_{1} u}{u_{3}}\right)=0$ & $\left(T_{12} u-T_{2} u\right) T_{1} u_{3}=\left(T_{1} u-u\right) T_{2} u_{3}$ \\
$K=\triangle_{1}\left(2 u_{3} \triangle_{2} u\right)+\partial_{3}\left(\left(\triangle_{1} u\right)^{2}-2 \triangle_{1} u \triangle_{2} u\right)=0$ & \\
\hline
\end{tabular}

\section{Case 5.}

\begin{tabular}{|l|l|}
\hline Conservation Laws & Differential-difference equation \\
\hline$I=\triangle_{1}\left(e^{\triangle_{2} u} u_{3}\right)+\partial_{3}\left(e^{\triangle_{2} u-\triangle_{1} u}-e^{\triangle_{2} u}\right)=0$ & $v\left(T_{12} v-T_{2} v\right) T_{1} v_{3}=T_{1} v\left(T_{1} v-v\right) T_{2} v_{3}$ \\
$J=\triangle_{1} \ln u_{3}+\triangle_{2} \ln \left(\frac{1-e^{\triangle_{1} u}}{u_{3}}\right)=0$ & $\left(\right.$ setting $\left.v=e^{u / \epsilon}\right)$ \\
$K=\triangle_{2}\left(\frac{u_{3}}{1-e^{\Delta_{1} u}}\right)+\partial_{3}\left(\ln \left(1-e^{\triangle_{1} u}\right)-\triangle_{1} u\right)=0$ & \\
\hline
\end{tabular}

\section{Case 6.}




\begin{tabular}{|l|l|}
\hline Conservation Laws & Differential-difference equation \\
\hline$I=\triangle_{1} \ln \left(\frac{\triangle_{2} u}{u_{3}}\right)+\triangle_{2} \ln \left(\frac{u_{3}}{\triangle_{1} u}\right)=0$ & $\left(T_{2} \triangle_{1} u\right)\left(\triangle_{2} u\right) T_{1} u_{3}=$ \\
$J=\triangle_{1}\left(\frac{u_{3}}{\triangle_{2} u}\right)+\partial_{3} \ln \left(1-\frac{\triangle_{1} u}{\triangle_{2} u}\right)=0$ & $\left(T_{1} \triangle_{2} u\right)\left(\triangle_{1} u\right) T_{2} u_{3}$ \\
$K=\triangle_{2}\left(\frac{u_{3}}{\triangle_{1} u}\right)+\partial_{3} \ln \left(1-\frac{\triangle_{2} u}{\triangle_{1} u}\right)=0$ & \\
\hline
\end{tabular}

\section{Case 7.}

\begin{tabular}{|l|l|}
\hline Conservation Laws & Differential-difference equation \\
\hline$I=\triangle_{1} \ln \left(\frac{\sinh \triangle_{2} u}{u_{3}}\right)-\triangle_{2} \ln \left(\frac{\sinh \triangle_{1} u}{u_{3}}\right)=0$ & $\left(T_{2} \sinh \triangle_{1} u\right)\left(\sinh \triangle_{2} u\right) T_{1} u_{3}=$ \\
$J=\triangle_{1}\left(u_{3} \operatorname{coth} \triangle_{2} u\right)+\triangle_{2} \ln \left(\frac{\sinh \left(\triangle_{1} u-\triangle_{2} u\right)}{\sinh \triangle_{2} u}\right)=0$ & $\left(T_{1} \sinh \triangle_{2} u\right)\left(\sinh \triangle_{1} u\right) T_{2} u_{3}$ \\
$K=\triangle_{2}\left(u_{3} \operatorname{coth} \triangle_{1} u\right)+\partial_{3} \ln \left(\frac{\sinh \left(\triangle_{1} u-\triangle_{2} u\right)}{\sinh \triangle_{1} u}\right)=0$ & \\
\hline
\end{tabular}

Remark. See the proof below for Lax pairs of the above differential-difference equations.

\section{Proof of Theorem 2:}

The proof is parallel to that of Theorem 1. The dispersionless limit of (15) is again a quasilinear conservation law of the form (9),

$$
\partial_{1} f+\partial_{2} g+\partial_{3} h=0
$$

where $f, g, h$ are functions of the variables $a=u_{1}, b=u_{2}, c=u_{3}$. Requiring that all one-phase reductions of the dispersionless equation are inherited by the differential-difference equation (15), we obtain a set of differential constraints for $f, g, h$ that are the necessary conditions for integrability. Thus, at the order $\epsilon$ we get

$$
f_{a}=g_{b}=h_{c}=0, \quad f_{c}+h_{a}+g_{c}+h_{b}=0,
$$

note the difference with Theorem 1 . The first set of these relations implies that the quasilinear conservation law is equivalent to the second-order equation

$$
F u_{12}+G u_{13}+H u_{23}=0,
$$

where $F=f_{b}+g_{a}, G=f_{c}+h_{a}, H=g_{c}+h_{b}$. Note that, by virtue of (16), the coefficients $F, G, H$ satisfy the additional constraint $G+H=0$. It follows from [9] that, up to a non-zero factor, any integrable equation of this type is equivalent to

$$
\left[p\left(u_{1}\right)-q\left(u_{2}\right)\right] u_{12}+r\left(u_{3}\right) u_{13}-r\left(u_{3}\right) u_{23}=0,
$$

where the functions $p(a), q(b), r(c)$ satisfy the integrability conditions

$$
\begin{gathered}
p^{\prime \prime}=p^{\prime}\left(\frac{p^{\prime}-q^{\prime}}{p-q}+(p-q) \frac{r^{\prime}}{r^{2}}\right), \\
q^{\prime \prime}=q^{\prime}\left(\frac{p^{\prime}-q^{\prime}}{p-q}-(p-q) \frac{r^{\prime}}{r^{2}}\right), \\
r^{\prime \prime}=2 \frac{r^{2}}{r} .
\end{gathered}
$$

Our further strategy is the same as in Theorem 1, namely: 
Step 1. First, we solve equations (18). Modulo unessential translations and rescalings this leads to seven quasilinear integrable equations of the form (17).

Step 2. For all of the seven equations found at step 1, we calculate first-order conservation laws (there will be four of them in each case).

Step 3. Taking linear combinations of the four conservation laws, and replacing $u_{1}, u_{2}$ by $\triangle_{1} u, \triangle_{2} u$ (keeping $u_{3}$ as it is), we obtain differential-difference equations (15) which are the candidates for integrability.

Step 4. Applying the $\epsilon^{2}$-integrability test, we find that only linear combinations of three conservation laws (out of four) pass the integrability test. Below we list conservation laws in such a way that the first three are the ones that pass the integrability test, while the fourth one doesn't. Moreover, each triplet of conservation laws corresponds to one and the same differential-difference equation.

Let us begin with the solution of system (18). The analysis leads to seven essentially different cases, which correspond to cases 1-7 of Theorem 2 in the same order as they appear below. First of all, the equation for $r$ implies that there are two essentially different cases: $r=1$ and $r=1 / c$.

Case 1: $r=1$. Then equations (18) simplify to

$$
p^{\prime \prime}=p^{\prime} \frac{p^{\prime}-q^{\prime}}{p-q}, \quad q^{\prime \prime}=q^{\prime} \frac{p^{\prime}-q^{\prime}}{p-q} .
$$

There are two subcases depending on how many functions among $p, q$ are constant.

subcase 1a: $q$ is constant (the case $p=$ const is similar). Without any loss of generality one can set $q=0$. Modulo unessential translations and rescalings this leads to $p=e^{a}$, resulting in the PDE

$$
e^{u_{1}} u_{12}+u_{13}-u_{23}=0
$$

This equation possesses four conservation laws:

$$
\begin{gathered}
\partial_{1} e^{u_{2}}-\partial_{3} e^{u_{2}-u_{1}}=0, \\
\partial_{1} u_{3}+\partial_{2}\left(e^{u_{1}}-u_{3}\right)=0, \\
\partial_{1} u_{3}^{2}+\partial_{2}\left(2 u_{3} e^{u_{1}}-e^{2 u_{1}}-u_{3}^{2}\right)-\partial_{3}\left(2 e^{u_{1}}\right)=0, \\
\partial_{1}\left(u_{2} u_{3}\right)+\partial_{2}\left(2 u_{1} e^{u_{1}}-2 e^{u_{1}}-u_{1} u_{3}\right)+\partial_{3}\left(u_{1}^{2}-u_{1} u_{2}\right)=0 .
\end{gathered}
$$

Applying steps 3 and 4 , we can show that semi-discrete versions of the first three conservation laws correspond to the differential-difference equation

$$
e^{\left(T_{12} u-T_{2} u\right) / \epsilon}-e^{\left(T_{1} u-u\right) / \epsilon}+T_{1} u_{3}-T_{2} u_{3}=0,
$$

which possesses the Lax pair

$$
T_{2} \psi=e^{\left(T_{1} u-T_{2} u\right) / \epsilon}\left(T_{1} \psi+\psi\right), \quad \epsilon \psi_{3}=-e^{\left(T_{1} u-u\right) / \epsilon}\left(T_{1} \psi+\psi\right) .
$$

Setting $v=e^{u / \epsilon}$ and $\partial_{3} \rightarrow \frac{1}{\epsilon} \partial_{3}$, we can rewrite (19) in the form

$$
\frac{T_{12} v}{T_{2} v}+\frac{T_{1} v_{3}}{T_{1} v}=\frac{T_{1} v}{v}+\frac{T_{2} v_{3}}{T_{2} v} .
$$


subcase 1b: both $p$ and $q$ are non-constant. Modulo unessential translations and rescalings, the elementary separation of variables gives $p=e^{a}, q=e^{b}$. The corresponding PDE is

$$
\left(e^{u_{1}}-e^{u_{2}}\right) u_{12}+u_{13}-u_{23}=0 .
$$

It possesses four conservation laws:

$$
\begin{gathered}
\partial_{1}\left(e^{u_{2}}-u_{3}\right)+\partial_{3} \ln \left(e^{u_{1}}-e^{u_{2}}\right)=0 \\
\partial_{2}\left(e^{u_{1}}-u_{3}\right)+\partial_{3} \ln \left(e^{u_{1}}-e^{u_{2}}\right)=0 \\
\partial_{1}\left(e^{2 u_{2}}-2 e^{u_{2}} u_{3}+u_{3}^{2}\right)+\partial_{2}\left(2 e^{u_{1}} u_{3}-e^{2 u_{1}}-u_{3}^{2}\right)+\partial_{3}\left(2 e^{u_{2}}-2 e^{u_{1}}\right)=0 \\
\partial_{1}\left(-2 e^{u_{2}} u_{2}+u_{2} u_{3}+2 e^{u_{2}}\right)+\partial_{2}\left(2 e^{u_{1}} u_{1}-u_{1} u_{3}-2 e^{u_{1}}\right)+ \\
\partial_{3}\left(u_{1} u_{2}-u_{2}^{2}+2\left(u_{1}-u_{2}\right) \ln \left(1-e^{u_{1}-u_{2}}\right)+2 L i_{2}\left(e^{u_{1}-u_{2}}\right)\right)=0 .
\end{gathered}
$$

Applying steps 3 and 4, we can show that semi-discrete versions of the first three conservation laws correspond to the differential-difference equation

$$
e^{\left(T_{12} u-T_{2} u\right) / \epsilon}-e^{\left(T_{12} u-T_{1} u\right) / \epsilon}+e^{\left(T_{2} u-u\right) / \epsilon}-e^{\left(T_{1} u-u\right) / \epsilon}+T_{1} u_{3}-T_{2} u_{3}=0 .
$$

Equation (20) possesses the Lax pair

$$
T_{2} \psi=e^{\left(T_{1} u-T_{2} u\right) / \epsilon} T_{1} \psi+\left(1-e^{\left(T_{1} u-T_{2} u\right) / \epsilon}\right) \psi, \quad \epsilon \psi_{3}=e^{\left(T_{1} u-u\right) / \epsilon}\left(T_{1} \psi-\psi\right) .
$$

Note that this case has been recorded before. Setting $v=e^{u / \epsilon}$ and $\partial_{3} \rightarrow \frac{1}{\epsilon} \partial_{3}$, we obtain the equation

$$
T_{12} v=\frac{T_{1} v T_{2} v}{v}+\frac{T_{2} v T_{1} v_{3}-T_{1} v T_{2} v_{3}}{T_{2} v-T_{1} v}
$$

which has appeared in the context of discrete evolutions of plane curves [1].

Case 2: $r=1 / c$. In this case the equations for $p$ and $q$ simplify to

$$
p^{\prime \prime}=p^{\prime}\left(\frac{p^{\prime}-q^{\prime}}{p-q}-(p-q)\right), \quad q^{\prime \prime}=q^{\prime}\left(\frac{p^{\prime}-q^{\prime}}{p-q}+(p-q)\right) .
$$

There are several subcases depending on how many functions among $p, q$ are constant.

subcase 2a: both $p$ and $q$ are constant. The corresponding PDE is

$$
u_{12}+\frac{1}{u_{3}}\left(u_{13}-u_{23}\right)=0 .
$$

It possesses four conservation laws:

$$
\begin{gathered}
\partial_{1}\left(e^{u_{2}} u_{3}\right)-\partial_{3} e^{u_{2}}=0, \\
\partial_{2}\left(e^{-u_{1}} u_{3}\right)+\partial_{3} e^{-u_{1}}=0, \\
\partial_{1}\left(u_{2}+\ln u_{3}\right)-\partial_{2} \ln u_{3}=0, \\
\partial_{1}\left(u_{2} u_{3}+2 u_{3}\right)+\partial_{2}\left(u_{1} u_{3}-2 u_{3}\right)-\partial_{3}\left(u_{1} u_{2}\right)=0 .
\end{gathered}
$$


Applying steps 3 and 4, we can show that semi-discrete versions of the first three conservation laws correspond to the differential-difference equation

$$
\frac{T_{2} u_{3}}{T_{1} u_{3}}=e^{\left(T_{12} u-T_{1} u-T_{2} u+u\right) / \epsilon} .
$$

This equation possesses the Lax pair

$$
T_{1} \psi=-e^{\left(T_{1} u-u\right) / \epsilon}\left(T_{2} \psi-\psi\right), \quad \epsilon \psi_{3}=-u_{3}\left(T_{2} \psi-\psi\right) .
$$

Setting $v=e^{u / \epsilon}$ we can rewrite $(21)$ as

$$
\frac{v T_{12} v}{T_{1} v}=\frac{T_{1} v T_{2} v_{3}}{T_{1} v_{3}} .
$$

subcase $\mathbf{2 b}: q$ is constant (the case $p=$ const is similar). Without any loss of generality one can set $q=0$. The equation for $p$ takes the form $p^{\prime \prime}=p^{2} / p-p p^{\prime}$, which integrates to $p^{\prime} / p+p=\alpha$. There are further subcases depending on the value of the integration constant $\alpha$.

subcase $2 \mathbf{b}(\mathbf{i}): \alpha=0$. Then one can take $p=1 / a$, which results in the PDE

$$
\frac{1}{u_{1}} u_{12}+\frac{1}{u_{3}}\left(u_{13}-u_{23}\right)=0 .
$$

It possesses four conservation laws:

$$
\begin{gathered}
\partial_{2}\left(u_{3} / u_{1}\right)-\partial_{3} \ln u_{1}=0, \\
\partial_{1} \ln u_{3}+\partial_{2} \ln \left(u_{1} / u_{3}\right)=0, \\
\partial_{1}\left(2 u_{2} u_{3}\right)+\partial_{3}\left(u_{1}^{2}-2 u_{1} u_{2}\right)=0, \\
\partial_{1}\left(u_{2}^{2} u_{3}\right)-\partial_{2}\left(\frac{u_{1}^{2} u_{3}}{3}\right)+\partial_{3}\left(u_{1}^{2} u_{2}-u_{2}^{2} u_{1}-\frac{2 u_{1}^{3}}{9}\right)=0 .
\end{gathered}
$$

Applying steps 3 and 4, we can show that semi-discrete versions of the first three conservation laws correspond to the differential-difference equation

$$
\left(T_{12} u-T_{2} u\right) T_{1} u_{3}=\left(T_{1} u-u\right) T_{2} u_{3} .
$$

This equation possesses the Lax pair

$$
T_{1} \psi=-\frac{\left(T_{1} u-u\right)}{\epsilon} T_{2} \psi+\psi, \quad \epsilon \psi_{3}=-u_{3} T_{2} \psi .
$$

subcase $2 \mathbf{b}(\mathbf{i i}): \alpha \neq 0$ (without any loss of generality one can set $\alpha=1$ ). Then one has $p=e^{a} /\left(e^{a}-1\right)$, which corresponds to the PDE

$$
\frac{e^{u_{1}}}{e^{u_{1}}-1} u_{12}+\frac{1}{u_{3}}\left(u_{13}-u_{23}\right)=0 .
$$

It possesses four conservation laws:

$$
\partial_{1}\left(u_{3} e^{u_{2}}\right)+\partial_{3}\left(e^{u_{2}-u_{1}}-e^{u_{2}}\right)=0,
$$




$$
\begin{gathered}
\partial_{1} \ln u_{3}+\partial_{2} \ln \left(\frac{1-e^{u_{1}}}{u_{3}}\right)=0 \\
\partial_{2}\left(\frac{u_{3}}{1-e^{u_{1}}}\right)+\partial_{3}\left(\ln \left(1-e^{u_{1}}\right)-u_{1}\right)=0 \\
\partial_{1}\left(\frac{u_{2} u_{3}}{2}+u_{3}\right)+\partial_{2}\left(\frac{u_{1} u_{3}\left(e^{u_{1}}+1\right)}{2\left(e^{u_{1}}-1\right)}-u_{3}\right)+\partial_{3}\left(\frac{u_{1}^{2}-u_{1} u_{2}}{2}-u_{1} \ln \left(1-e^{u_{1}}\right)-L i_{2}\left(e^{u_{1}}\right)\right)=0 .
\end{gathered}
$$

Applying steps 3 and 4, we can show that semi-discrete versions of the first three conservation laws correspond to the differential-difference equation

$$
\left(1-e^{\left(T_{12} u-T_{2} u\right) / \epsilon}\right) T_{1} u_{3}=\left(1-e^{\left(T_{1} u-u\right) / \epsilon}\right) T_{2} u_{3},
$$

which possesses the Lax pair

$$
T_{1} \psi=\left(1-e^{\left(T_{1} u-u\right) / \epsilon}\right) T_{2} \psi-e^{\left(T_{1} u-u\right) / \epsilon} \psi, \quad \epsilon \psi_{3}=u_{3} T_{2} \psi+u_{3} \psi .
$$

Setting $v=e^{u / \epsilon}$ we can rewrite equation (23) in the form

$$
v\left(T_{12} v-T_{2} v\right) T_{1} v_{3}=T_{1} v\left(T_{1} v-v\right) T_{2} v_{3} .
$$

subcase 2c: both $p$ and $q$ are non-constant. Subtracting the ODEs for $p$ and $q$ from each other and separating the variables gives $p^{\prime}=\alpha-p^{2}, q^{\prime}=\alpha-q^{2}$. There are further subcases depending on the value of the integration constant $\alpha$.

subcase 2c(i): $\alpha=0$. Then one can take $p=1 / a, q=1 / b$, which results in the PDE

$$
\left(\frac{1}{u_{1}}-\frac{1}{u_{2}}\right) u_{12}+\frac{1}{u_{3}}\left(u_{13}-u_{23}\right)=0 .
$$

It possesses four conservation laws:

$$
\begin{gathered}
\partial_{1} \ln \left(\frac{u_{2}}{u_{3}}\right)+\partial_{2} \ln \left(\frac{u_{3}}{u_{1}}\right)=0, \\
\partial_{1}\left(\frac{u_{3}}{u_{2}}\right)+\partial_{3} \ln \left(1-\frac{u_{1}}{u_{2}}\right)=0, \\
\partial_{2}\left(\frac{u_{3}}{u_{1}}\right)+\partial_{3} \ln \left(1-\frac{u_{2}}{u_{1}}\right)=0, \\
\partial_{1}\left(u_{2}^{2} u_{3}\right)-\partial_{2}\left(u_{1}^{2} u_{3}\right)+\partial_{3}\left(u_{1}^{2} u_{2}-u_{2}^{2} u_{1}\right)=0 .
\end{gathered}
$$

Applying steps 3 and 4, we can show that semi-discrete versions of the first three conservation laws correspond to the differential-difference equation

$$
\left(T_{2} \triangle_{1} u\right)\left(\triangle_{2} u\right) T_{1} u_{3}=\left(T_{1} \triangle_{2} u\right)\left(\triangle_{1} u\right) T_{2} u_{3},
$$

which appeared in [7]. Equation (24) possesses the Lax pair

$$
T_{1} \psi=\frac{\triangle_{1} u}{\triangle_{2} u} T_{2} \psi+\left(1-\frac{\triangle_{1} u}{\triangle_{2} u}\right) \psi, \quad \epsilon \psi_{3}=\frac{u_{3}}{\triangle_{2} u}\left(T_{2} \psi-\psi\right) .
$$


subcase 2c(ii): $\alpha \neq 0$ (we will consider the hyperbolic case $\alpha=1$; the trigonometric case $\alpha=-1$ is similar). Then one can take $p=\operatorname{coth} a, q=\operatorname{coth} b$, which results in the PDE

$$
\left(\operatorname{coth} u_{1}-\operatorname{coth} u_{2}\right) u_{12}+\frac{1}{u_{3}}\left(u_{13}-u_{23}\right)=0 .
$$

It possesses four conservation laws:

$$
\begin{gathered}
\partial_{1} \ln \left(\frac{\sinh u_{2}}{u_{3}}\right)-\partial_{2} \ln \left(\frac{\sinh u_{1}}{u_{3}}\right)=0, \\
\partial_{1}\left(u_{3} \operatorname{coth} u_{2}\right)+\partial_{2} \ln \left(\frac{\sinh \left(u_{1}-u_{2}\right)}{\sinh u_{2}}\right)=0, \\
\partial_{2}\left(u_{3} \operatorname{coth} u_{1}\right)+\partial_{3} \ln \left(\frac{\sinh \left(u_{1}-u_{2}\right)}{\sinh u_{1}}\right)=0, \\
\partial_{1}\left(4 u_{3}\left(1-\operatorname{coth} u_{2}-u_{2} \operatorname{coth} u_{2}\right)\right)+\partial_{2}\left(4 u_{1} u_{3} \operatorname{coth} u_{1}\right)+ \\
\partial_{3}\left(4 u_{1}-2 u_{2}^{2}-12 u_{2}+2\left(u_{1}-u_{2}-2\right) \ln \left(1-e^{2 u_{1}-2 u_{2}}\right)+2\left(u_{1}-u_{2}\right) \ln \left(1-e^{2 u_{2}-2 u_{1}}\right)+\right. \\
4\left(u_{2}+1\right) \ln \left(1-e^{2 u_{2}}\right)-2 u_{1} \ln \left(\left(1-e^{-2 u_{1}}\right)\left(1-e^{2 u_{1}}\right)\right)+L i_{2}\left(e^{-2 u_{1}}\right)-L i_{2}\left(e^{2 u_{1}}\right)+ \\
\left.L i_{2}\left(e^{2 u_{1}-2 u_{2}}\right)+2 L i_{2}\left(e^{2 u_{2}}\right)-L i_{2}\left(e^{2 u_{2}-2 u_{1}}\right)\right)=0 .
\end{gathered}
$$

Applying steps 3 and 4, we can show that semi-discrete versions of the first three conservation laws correspond to the differential-difference equation

$$
\left(T_{2} \sinh \triangle_{1} u\right)\left(\sinh \triangle_{2} u\right) T_{1} u_{3}=\left(T_{1} \sinh \triangle_{2} u\right)\left(\sinh \triangle_{1} u\right) T_{2} u_{3},
$$

which possesses the following Lax pair:

$$
T_{2} \psi=\frac{e^{2 \triangle_{2} u}-1}{e^{2 \triangle_{1} u}-1} T_{1} \psi+\frac{e^{2 \triangle_{1} u}-e^{2 \triangle_{2} u}}{e^{2 \triangle_{1} u}-1} \psi, \quad \epsilon \psi_{3}=\frac{2 u_{3}}{e^{2 \triangle_{1} u}-1}\left(T_{1} \psi-\psi\right) .
$$

This finishes the proof of Theorem 2 .

\subsection{One discrete and two continuous variables.}

One can show that there exist no non-degenerate integrable equations of the form

$$
\triangle_{1} f+\partial_{2} g+\partial_{3} h=0,
$$

where $f, g, h$ are functions of $\triangle_{1} u, u_{2}, u_{3}$.

\section{Discrete second-order quasilinear equations in 3D}

Here we present the result of classification of integrable equations of the form

$$
\sum_{i, j=1}^{3} f_{i j}(\triangle u) \triangle_{i j} u=0,
$$


where $f_{i j}$ are functions of $\triangle_{1} u, \triangle_{2} u, \triangle_{3} u$ only. These equations can be viewed as discretisations of second-order quasilinear PDEs

$$
\sum_{i, j=1}^{3} f_{i j}\left(u_{k}\right) u_{i j}=0,
$$

whose integrability was investigated in [9].

Theorem 3 There exists a unique non-degenerate discrete second-order quasilinear equation in $3 D$, known as the lattice KP equation:

$$
\left(\triangle_{1} u-\triangle_{2} u\right) \triangle_{12} u+\left(\triangle_{3} u-\triangle_{1} u\right) \triangle_{13} u+\left(\triangle_{2} u-\triangle_{3} u\right) \triangle_{23} u=0 .
$$

In different contexts and equivalent forms, it has appeared in $[10,36,35]$. The proof is similar to that of Theorem 1, and will be omitted.

\subsection{Two discrete and one continuous variables}

The classification of semi-discrete integrable equations of the form

$$
f_{11} \triangle_{11} u+f_{12} \triangle_{12} u+f_{22} \triangle_{22} u+f_{13} \triangle_{1} u_{3}+f_{23} \triangle_{2} u_{3}+f_{33} u_{33}=0,
$$

where the coefficients $f_{i j}$ are functions of $\left(\triangle_{1} u, \triangle_{2} u, u_{3}\right)$, gives the following result:

Theorem 4 There exists a unique non-degenerate second-order equations of the above type, known as the semi-discrete Toda lattice,

$$
\left(\triangle_{1} u-\triangle_{2} u\right) \triangle_{12} u-\triangle_{1} u_{3}+\triangle_{2} u_{3}=0 .
$$

It has appeared before in $[1,31]$. Again, we skip the details of calculations.

\subsection{One discrete and two continuous variables}

One can show that there exist no non-degenerate semi-discrete integrable equations of the form

$$
f_{11} \triangle_{11} u+f_{12} \triangle_{1} u_{2}+f_{22} u_{22}+f_{13} \triangle_{1} u_{3}+f_{23} u_{23}+f_{33} u_{33}=0
$$

where the coefficients $f_{i j}$ are functions of $\left(\triangle_{1} u, u_{2}, u_{3}\right)$.

\section{$5 \quad$ Numerics}

In this section we compare numerical solutions for the discrete equation (7),

$$
\triangle_{t \bar{t}} u-\triangle_{x \bar{x}}\left[u-\ln \left(e^{u}+1\right)\right]-\triangle_{y \bar{y}}\left[\ln \left(e^{u}+1\right)\right]=0,
$$

and its dispersionless limit (8),

$$
u_{t t}-\left[u-\ln \left(e^{u}+1\right)\right]_{x x}-\left[\ln \left(e^{u}+1\right)\right]_{y y}=0,
$$

obtained using Mathematica. We choose the following Cauchy data: 
Discrete equation (7): $u(x, y, 0)=3 e^{-\left(x^{2}+y^{2}\right)}, u(x, y,-\epsilon)=3 e^{-\left(x^{2}+y^{2}\right)}$.

Dispersionless equation (8): $u(x, y, 0)=3 e^{-\left(x^{2}+y^{2}\right)}, u_{t}(x, y, 0)=0$.

In Figure 1 we plot the numerical solution of the dispersionless equation (8) for $t=0,4,8$. As equation (8) does not satisfy the null conditions of Klainerman [24], according to the general theory this solution is expected to break down in finite time.
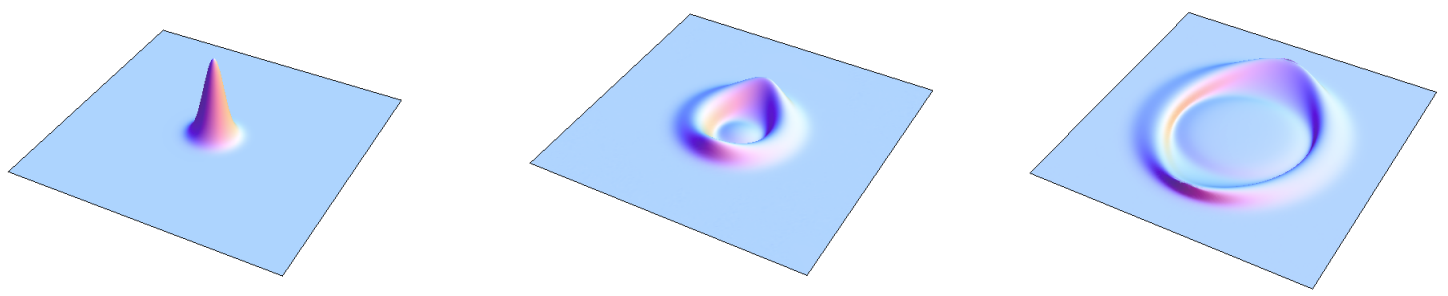

Figure 1: Numerical solution of the dispersionless equation (8) for $t=0,4,8$, showing the onset of breaking.

On the contrary, solutions to the dispersive regularisation (7) (which can be viewed as a difference scheme) do not break down. Indeed, (7) can be rewritten in the form

$$
u(t+\epsilon)=-u(t-\epsilon)+\left(T_{x}+T_{\bar{x}}\right)\left(u-\ln \left(e^{u}+1\right)\right)+\left(T_{y}+T_{\bar{y}}\right) \ln \left(e^{u}+1\right),
$$

which allows the computation of $u(t+\epsilon)$ once $u(t)$ and $u(t-\epsilon)$ are known. Figures 2, 3 and 4 illustrate the solution for different values of $\epsilon$ at $t=0,4,8$. As $\epsilon$ becomes smaller one can see the formation of a dispersive shock wave in Figure 5.
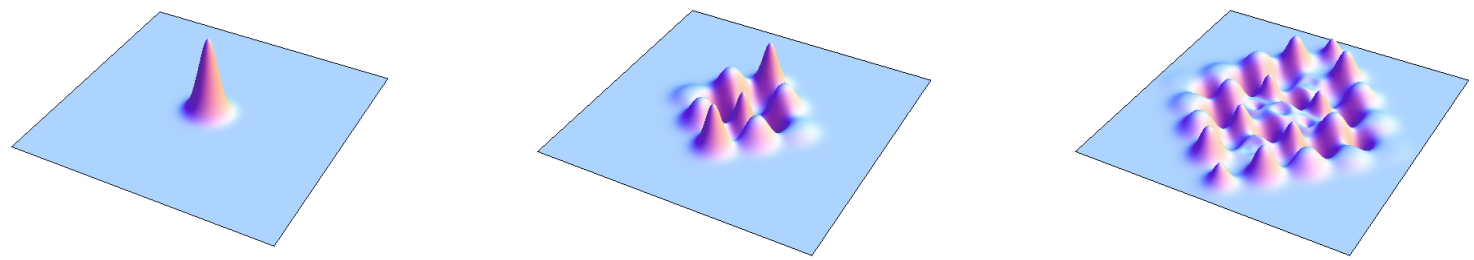

Figure 2: Solution of the discrete equation (7) for $\epsilon=2$ and $t=0,4,8$.
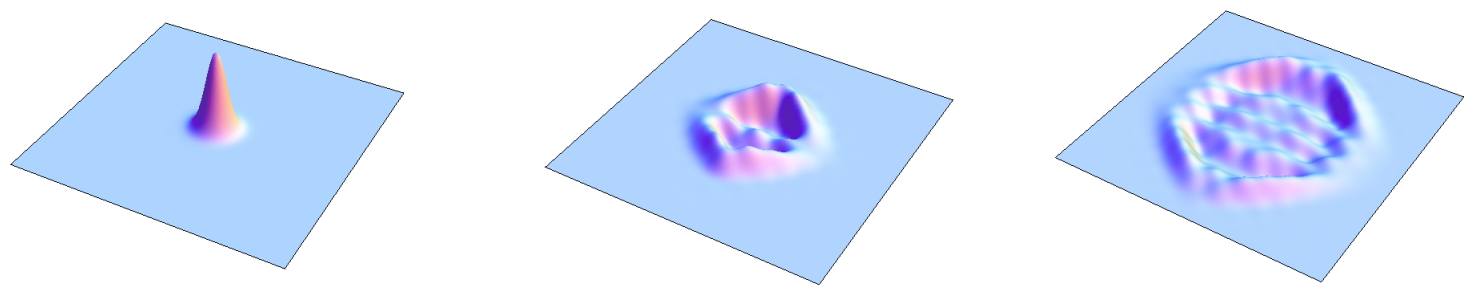

Figure 3: Solution of the discrete equation (7) for $\epsilon=1$ and $t=0,4,8$. 

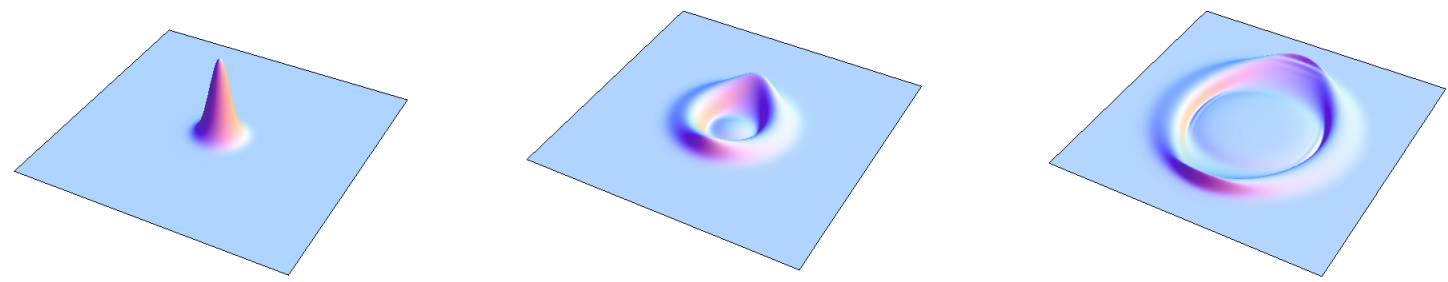

Figure 4: Solution of the discrete equation (7) for $\epsilon=1 / 8$ and $t=0,4,8$. As $\epsilon \rightarrow 0$, solutions of the discrete equation tend to solutions of the dispersionless limit until the breakdown occurs. At the breaking point, one can see the formation of a dispersive shock wave.
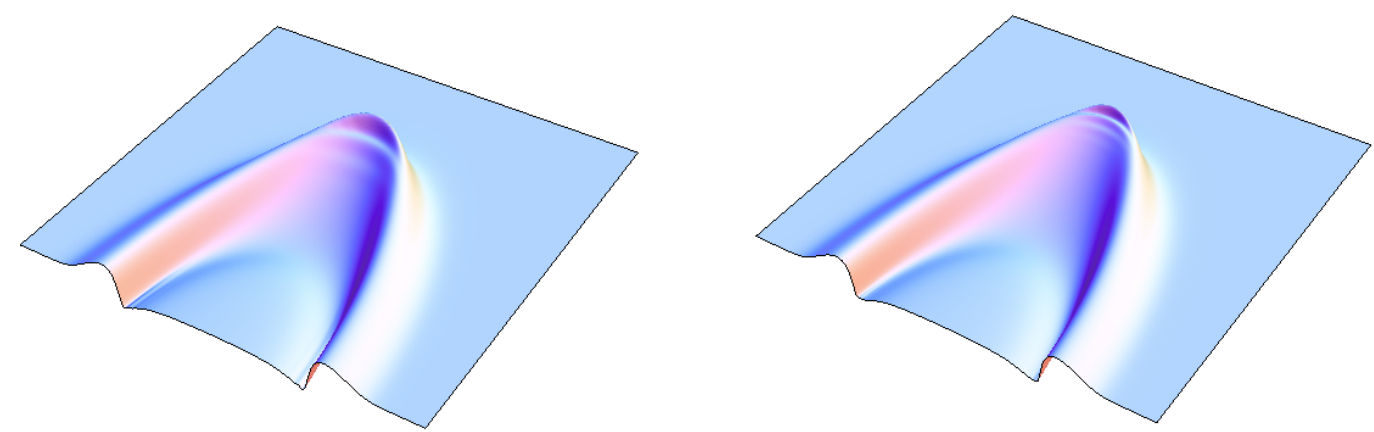

Figure 5: Formation of a dispersive shock wave in the solution of the discrete equation (7) for $\epsilon=1 / 8$ (left) and $\epsilon=1 / 16$ (right), at $t=8$.

We would like to emphasize that there are very few results on dispersive shock waves in $2+1$ dimensions (see $[25,26]$ for a detailed numerical investigation of this phenomenon for the KP and DS equations). This is primarily due to the computational complexity of problems involving rapid oscillations. On the contrary, in the discrete example discussed in this section one does not require dedicated numerical methods to observe the formation of a dispersive shock wave: this is achieved by simply iterating an explicit recurrence relation (26). We hope that this example will be useful for the general theory of dispersive shock waves in higher dimensions (yet to be developed).

\section{Appendix: $\triangle$-forms of Hirota-type difference equations}

Below we list $\triangle$-forms of various $3 \mathrm{D}$ discrete integrable equations that have been discussed in the literature. The advantage of $\triangle$-representation is that the corresponding dispersionless limits become more clearly seen. Although these equations have appeared under different names, most of them are related via various gauge/Miura/Bäcklund type transformations. We have verified that all equations listed below inherit hydrodynamic reductions of their dispersionless limits, at least to the order $\epsilon^{2}$.

Hirota equation [20]:

$$
\alpha T_{1} \tau T_{\overline{1}} \tau+\beta T_{2} \tau T_{\overline{2}} \tau+\gamma T_{3} \tau T_{\overline{3}} \tau=0 .
$$


Dividing by $\tau^{2}$ and setting $\tau=e^{u / \epsilon^{2}}$ we can rewrite it in the form

$$
\alpha e^{\triangle_{1 \overline{1}} u}+\beta e^{\triangle_{2 \overline{2}} u}+\gamma e^{\triangle_{3 \overline{3}} u}=0 .
$$

Its dispersionless limit is

$$
\alpha e^{u_{11}}+\beta e^{u_{22}}+\gamma e^{u_{33}}=0
$$

Hirota-Miwa equation [34]:

$$
\alpha T_{1} \tau T_{23} \tau+\beta T_{2} \tau T_{13} \tau+\gamma T_{3} \tau T_{12} \tau=0 .
$$

Dividing by $T_{1} \tau T_{2} \tau T_{3} \tau / \tau$ and setting $\tau=e^{u / \epsilon^{2}}$ we can rewrite it in the form

$$
\alpha e^{\triangle_{23} u}+\beta e^{\triangle_{13} u}+\gamma e^{\triangle_{12} u}=0 .
$$

Its dispersionless limit is

$$
\alpha e^{u_{23}}+\beta e^{u_{13}}+\gamma e^{u_{12}}=0
$$

Gauge-invariant Hirota equation, or Y-system [48, 29]:

$$
\frac{T_{2} v T_{\overline{2}} v}{T_{1} v T_{\overline{1}} v}=\frac{\left(1+T_{3} v\right)\left(1+T_{\overline{3}} v\right)}{\left(1+T_{1} v\right)\left(1+T_{\overline{1}} v\right)} .
$$

Taking $\log$ of both sides we obtain

$$
\left(\triangle_{2 \overline{2}}-\triangle_{1 \overline{1}}\right) \ln v=\left(\triangle_{3 \overline{3}}-\triangle_{1 \overline{1}}\right) \ln (v+1) .
$$

Setting $v=e^{u}$ we get

$$
\triangle_{2 \overline{2}} u=\triangle_{1 \overline{1}}\left[u-\ln \left(e^{u}+1\right)\right]+\triangle_{3 \overline{3}}\left[\ln \left(e^{u}+1\right)\right]=0,
$$

its dispersionless limit is

$$
u_{22}=\left[u-\ln \left(e^{u}+1\right)\right]_{11}+\left[\ln \left(e^{u}+1\right)\right]_{33} .
$$

Lattice KP equation [10, 36, 35]:

$$
\left(T_{1} u-T_{2} u\right) T_{12} u+\left(T_{3} u-T_{1} u\right) T_{13} u+\left(T_{2} u-T_{3} u\right) T_{23} u=0 .
$$

In equivalent form,

$$
\left(\triangle_{1} u-\triangle_{2} u\right) \triangle_{12} u+\left(\triangle_{3} u-\triangle_{1} u\right) \triangle_{13} u+\left(\triangle_{2} u-\triangle_{3} u\right) \triangle_{23} u=0 .
$$

Its dispersionless limit is

$$
\left(u_{1}-u_{2}\right) u_{12}+\left(u_{3}-u_{1}\right) u_{13}+\left(u_{2}-u_{3}\right) u_{23}=0 .
$$

Schwarzian KP equation [36, 13, 6, 7, 27]:

$$
\left(T_{2} \triangle_{1} u\right)\left(T_{3} \triangle_{2} u\right)\left(T_{1} \triangle_{3} u\right)=\left(T_{2} \triangle_{3} u\right)\left(T_{3} \triangle_{1} u\right)\left(T_{1} \triangle_{2} u\right) .
$$


Taking log of both sides we obtain

$$
\triangle_{1}\left(\ln \frac{\triangle_{3} u}{\triangle_{2} u}\right)+\triangle_{2}\left(\ln \frac{\triangle_{1} u}{\triangle_{3} u}\right)+\triangle_{3}\left(\ln \frac{\triangle_{2} u}{\triangle_{1} u}\right)=0 .
$$

Its dispersionless limit is

$$
u_{3}\left(u_{2}-u_{1}\right) u_{12}+u_{2}\left(u_{1}-u_{3}\right) u_{13}+u_{1}\left(u_{3}-u_{2}\right) u_{23}=0 .
$$

\section{Lattice spin equation [37]:}

$$
\left(\frac{T_{12} \tau}{T_{2} \tau}-1\right)\left(\frac{T_{13} \tau}{T_{1} \tau}-1\right)\left(\frac{T_{23} \tau}{T_{3} \tau}-1\right)=\left(\frac{T_{12} \tau}{T_{1} \tau}-1\right)\left(\frac{T_{13} \tau}{T_{3} \tau}-1\right)\left(\frac{T_{23} \tau}{T_{2} \tau}-1\right)
$$

On multiplication by $T_{1} \tau T_{2} \tau T_{3} \tau$ it reduces to the Schwarzian KP equation. An alternative representation can be obtained by taking $\log$ of both sides and setting $\tau=e^{u / \epsilon}$. This gives

$$
\triangle_{1} \ln \frac{e^{\triangle_{3} u}-1}{e^{\triangle_{2} u}-1}+\triangle_{2} \ln \frac{e^{\triangle_{1} u}-1}{e^{\triangle_{3} u}-1}+\triangle_{3} \ln \frac{e^{\triangle_{2} u}-1}{e^{\triangle_{1} u}-1}=0 .
$$

Its dispersionless limit is

$$
\frac{e^{u_{2}}-e^{u_{1}}}{\left(e^{u_{1}}-1\right)\left(e^{u_{2}}-1\right)} u_{12}+\frac{e^{u_{1}}-e^{u_{3}}}{\left(e^{u_{1}}-1\right)\left(e^{u_{3}}-1\right)} u_{13}+\frac{e^{u_{3}}-e^{u_{2}}}{\left(e^{u_{2}}-1\right)\left(e^{u_{3}}-1\right)} u_{23}=0 .
$$

Sine-Gordon equation [27]:

$$
\left(T_{2} \sin \triangle_{1} u\right)\left(T_{3} \sin \triangle_{2} u\right)\left(T_{1} \sin \triangle_{3} u\right)=\left(T_{2} \sin \triangle_{3} u\right)\left(T_{3} \sin \triangle_{1} u\right)\left(T_{1} \sin \triangle_{2} u\right) .
$$

Taking log of both sides we obtain

$$
\triangle_{1}\left(\ln \frac{\sin \triangle_{3} u}{\sin \triangle_{2} u}\right)+\triangle_{2}\left(\ln \frac{\sin \triangle_{1} u}{\sin \triangle_{3} u}\right)+\triangle_{3}\left(\ln \frac{\sin \triangle_{2} u}{\sin \triangle_{1} u}\right)=0 .
$$

Its dispersionless limit is

$$
\left(\cot u_{2}-\cot u_{1}\right) u_{12}+\left(\cot u_{1}-\cot u_{3}\right) u_{13}+\left(\cot u_{3}-\cot u_{2}\right) u_{23}=0 .
$$

This example is nothing but trigonometric version of the lattice spin equation.

Lattice mKP equation [37]:

$$
\frac{T_{13} \tau-T_{12} \tau}{T_{1} \tau}+\frac{T_{12} \tau-T_{23} \tau}{T_{2} \tau}+\frac{T_{23} \tau-T_{13} \tau}{T_{3} \tau}=0 .
$$

Setting $\tau=e^{u / \epsilon}$ we obtain

$$
\triangle_{1}\left(e^{\triangle_{3} u}-e^{\triangle_{2} u}\right)+\triangle_{2}\left(e^{\triangle_{1} u}-e^{\triangle_{3} u}\right)+\triangle_{3}\left(e^{\triangle_{2} u}-e^{\triangle_{1} u}\right)=0,
$$

its dispersionless limit is

$$
\left(e^{u_{1}}-e^{u_{2}}\right) u_{12}+\left(e^{u_{3}}-e^{u_{1}}\right) u_{13}+\left(e^{u_{2}}-e^{u_{3}}\right) u_{23}=0 .
$$


Toda equation $[30,48]$ :

$$
\alpha T_{1} \tau T_{2} \tau+\beta \tau T_{12} \tau+\gamma T_{1 \overline{3}} \tau T_{23} \tau=0 .
$$

Dividing by $T_{1} \tau T_{2} \tau$ and setting $\tau=e^{u / \epsilon^{2}}$ we get

$$
\alpha+\beta e^{\triangle_{12} u}+\gamma e^{\triangle_{23} u-\triangle_{1 \overline{3}} u+\triangle_{3 \overline{3}} u}=0,
$$

its dispersionless limit is

$$
\alpha+\beta e^{u_{12}}+\gamma e^{u_{23}-u_{13}+u_{33}}=0 .
$$

Lattice Toda equation [37]:

$$
\left(T_{1}-T_{3}\right) \frac{T_{2} \tau}{\tau}=\left(T_{2}-T_{3}\right) \frac{T_{1} \tau}{\tau} .
$$

Setting $\tau=e^{u / \epsilon}$ we get

$$
\triangle_{1}\left(e^{\triangle_{2} u}\right)-\triangle_{2}\left(e^{\triangle_{1} u}\right)+\triangle_{3}\left(e^{\triangle_{1} u}-e^{\triangle_{2} u}\right)=0,
$$

its dispersionless limit is

$$
\left(e^{u_{2}}-e^{u_{1}}\right) u_{12}+e^{u_{1}} u_{13}-e^{u_{2}} u_{23}=0 .
$$

Lattice mToda equation [37]:

$$
\left(\frac{T_{13} \tau}{T_{1} \tau}-1\right)\left(\frac{T_{23} \tau}{T_{3} \tau}-1\right)=\left(\frac{T_{12} \tau}{T_{1} \tau}-1\right)\left(\frac{T_{23} \tau}{T_{2} \tau}-1\right)
$$

Taking $\log$ of both sides and setting $\tau=e^{u / \epsilon}$ we get

$$
\triangle_{1} \ln \frac{e^{\triangle_{3} u}-1}{e^{\triangle_{2} u}-1}-\triangle_{2} \ln \left(e^{\triangle_{3} u}-1\right)+\triangle_{3} \ln \left(e^{\triangle_{2} u}-1\right)=0 .
$$

Its dispersionless limit is

$$
-\frac{e^{u_{2}}}{e^{u_{2}}-1} u_{12}+\frac{e^{u_{3}}}{e^{u_{3}}-1} u_{13}+\frac{e^{u_{3}}-e^{u_{2}}}{\left(e^{u_{2}}-1\right)\left(e^{u_{3}}-1\right)} u_{23}=0 .
$$

Toda equation for rotation coefficients [11]:

$$
\left(T_{2}-1\right) \frac{T_{1} \tau}{\tau}=T_{1} \frac{T_{2} \tau}{T_{\overline{3}} \tau}-\frac{T_{23} \tau}{\tau} .
$$

This equation appeared in the theory of Laplace transformations of discrete quadrilateral nets. Setting $\tau=e^{u / \epsilon}$ we obtain

$$
\triangle_{2}\left(e^{\triangle_{1} u}\right)=\left(\triangle_{1}-\triangle_{3}\right) e^{\triangle_{2} u+\triangle_{\overline{3}} u} .
$$

Its dispersionless limit is

$$
e^{u_{1}} u_{12}=e^{u_{2}+u_{3}}\left(u_{12}+u_{13}-u_{23}-u_{33}\right) .
$$


One more version of the Toda equation [6]:

$$
T_{\overline{1} 3} \tau+\alpha T_{2} \tau=T_{\overline{1}} \tau T_{3} \tau\left(\frac{1}{\tau}+\alpha \frac{1}{T_{\overline{1} \overline{2} 3} \tau}\right)
$$

Setting $\tau=e^{-u / \epsilon}$ we obtain

$$
\triangle_{3} e^{\triangle_{\overline{1}} u}=\alpha\left(\epsilon \triangle_{\overline{1} \overline{2}}-\triangle_{\overline{1}}-\triangle_{\overline{2}}\right) e^{\triangle_{3} u-\triangle_{2} u} .
$$

Its dispersionless limit is

$$
e^{u_{1}} u_{13}+\alpha e^{u_{3}-u_{2}}\left(u_{13}+u_{23}-u_{12}-u_{22}\right)=0 .
$$

Schwarzian Toda equation [6, 7]:

$$
\left(T_{1} \triangle_{3} u\right)\left(T_{2}\left(\triangle_{1}+\triangle_{\overline{2}}\right) u\right)\left(T_{3} \triangle_{\overline{2}} u\right)=\left(\triangle_{3} u\right)\left(T_{3}\left(\triangle_{1}+\triangle_{\overline{2}}\right) u\right)\left(T_{1} \triangle_{2} u\right) .
$$

Taking log of both sides we obtain

$$
\triangle_{1} \ln \triangle_{3} u+\left(\triangle_{2}-\triangle_{3}\right) \ln \left(\triangle_{1}+\triangle_{\overline{2}}\right) u+\triangle_{3} \ln \triangle_{\overline{2}} u-\triangle_{1} \ln \triangle_{2} u+\frac{1}{\epsilon} \ln \left(1-\epsilon \frac{\triangle_{2 \overline{2}} u}{\triangle_{2} u}\right)=0
$$

Its dispersionless limit is

$$
\frac{u_{2}}{u_{1} u_{3}}\left(u_{1}+u_{2}-u_{3}\right) u_{13}-u_{12}-u_{22}+u_{23}=0
$$

BKP equation in Miwa form [34, 39]:

$$
\alpha T_{1} \tau T_{23} \tau+\beta T_{2} \tau T_{13} \tau+\gamma T_{3} \tau T_{12} \tau+\delta \tau T_{123} \tau=0
$$

This equation can be interpreted as the permutability theorem of Moutard transformations [39]. Dividing by $T_{1} \tau T_{2} \tau T_{3} \tau / \tau$ and setting $\tau=e^{u / \epsilon^{2}}$ we get

$$
\alpha e^{\triangle_{23} u}+\beta e^{\triangle_{13} u}+\gamma e^{\triangle_{12} u}+\delta e^{\epsilon \triangle_{123} u+\triangle_{23} u+\triangle_{13} u+\triangle_{12} u}=0 .
$$

Its dispersionless limit is

$$
\alpha e^{u_{23}}+\beta e^{u_{13}}+\gamma e^{u_{12}}+\delta e^{u_{23}+u_{13}+u_{12}}=0 .
$$

BKP equation in Hirota form [34]:

$$
\alpha T_{1} \tau T_{\overline{1}} \tau+\beta T_{2} \tau T_{\overline{2}} \tau+\gamma T_{3} \tau T_{\overline{3}} \tau+\delta T_{123} \tau T_{\overline{1} \overline{2} \overline{3}} \tau=0 .
$$

Dividing by $\tau^{2}$ and setting $\tau=e^{u / \epsilon^{2}}$ we get

$$
\alpha e^{\triangle_{1 \overline{1}} u}+\beta e^{\triangle_{2 \overline{2}} u}+\gamma e^{\triangle_{3 \overline{3}} u}+\delta e^{\epsilon\left(\triangle_{123} u-\triangle_{\overline{1} \overline{2} \overline{3}} u\right)+S}=0,
$$

where

$$
S=\left(\triangle_{1 \overline{1}} u+\triangle_{2 \overline{2}} u+\triangle_{3 \overline{3}} u\right)+\left(\triangle_{12} u+\triangle_{\overline{1} \overline{2}} u\right)+\left(\triangle_{13} u+\triangle_{\overline{1} \overline{3}} u\right)+\left(\triangle_{23} u+\triangle_{\overline{2} \overline{3}} u\right) .
$$


Its dispersionless limit is

$$
\alpha e^{u_{11}}+\beta e^{u_{22}}+\gamma e^{u_{33}}+\delta e^{u_{11}+u_{22}+u_{33}+2 u_{12}+2 u_{13}+2 u_{23}}=0 .
$$

Schwarzian BKP equation [40, 28, 43]:

$$
\frac{\left(T_{1} u-T_{2} u\right)\left(T_{123} u-T_{3} u\right)}{\left(T_{2} u-T_{3} u\right)\left(T_{123} u-T_{1} u\right)}=\frac{\left(T_{13} u-T_{23} u\right)\left(T_{12} u-u\right)}{\left(T_{12} u-T_{13} u\right)\left(T_{23} u-u\right)} .
$$

Taking log of both sides we get

$$
\triangle_{3} \ln \frac{\epsilon \triangle_{12} u+\triangle_{1} u+\triangle_{2} u}{\triangle_{1} u-\triangle_{2} u}=\triangle_{1} \ln \frac{\epsilon \triangle_{23} u+\triangle_{2} u+\triangle_{3} u}{\triangle_{3} u-\triangle_{2} u} .
$$

Its dispersionless limit is [8]:

$$
u_{3}\left(u_{2}^{2}-u_{1}^{2}\right) u_{12}+u_{2}\left(u_{1}^{2}-u_{3}^{2}\right) u_{13}+u_{1}\left(u_{3}^{2}-u_{2}^{2}\right) u_{23}=0 .
$$

It was shown in [43] that the Schwarzian BKP equation is the only non-linearizable affine linear discrete equation consistent around a $4 \mathrm{D}$ cube.

BKP version of the sine-Gordon equation [40, 28]:

$$
\frac{\sin \left(T_{1} u-T_{2} u\right) \sin \left(T_{123} u-T_{3} u\right)}{\sin \left(T_{2} u-T_{2} u\right) \sin \left(T_{123} u-T_{1} u\right)}=\frac{\sin \left(T_{13} u-T_{23} u\right) \sin \left(T_{12} u-u\right)}{\sin \left(T_{12} u-T_{13} u\right) \sin \left(T_{23} u-u\right)} .
$$

Taking log of both sides we get

$$
\triangle_{3} \ln \frac{\sin \left(\epsilon \triangle_{12} u+\triangle_{1} u+\triangle_{2} u\right)}{\sin \left(\triangle_{1} u-\triangle_{2} u\right)}=\triangle_{1} \ln \frac{\sin \left(\epsilon \triangle_{23} u+\triangle_{2} u+\triangle_{3} u\right)}{\sin \left(\triangle_{3} u-\triangle_{2} u\right)} .
$$

Its dispersionless limit is

$$
\begin{gathered}
\sin 2 u_{3}\left(\sin ^{2} u_{2}-\sin ^{2} u_{1}\right) u_{12}+\sin 2 u_{2}\left(\sin ^{2} u_{1}-\sin ^{2} u_{3}\right) u_{13}+ \\
\sin 2 u_{1}\left(\sin ^{2} u_{3}-\sin ^{2} u_{2}\right) u_{23}=0 .
\end{gathered}
$$

CKP equation [41]:

$$
\begin{gathered}
\left(\tau T_{123} \tau-T_{1} \tau T_{23} \tau-T_{2} \tau T_{13} \tau-T_{3} \tau T_{12} \tau\right)^{2}= \\
4\left(T_{1} \tau T_{2} \tau T_{13} \tau T_{23} \tau+T_{2} \tau T_{3} \tau T_{12} \tau T_{13} \tau+T_{1} \tau T_{3} \tau T_{12} \tau T_{23} \tau-T_{1} \tau T_{2} \tau T_{3} \tau T_{123} \tau-\tau T_{12} \tau T_{13} \tau T_{23} \tau\right) .
\end{gathered}
$$

Multiplying by $\left[\tau /\left(T_{1} \tau T_{2} \tau T_{3} \tau\right)\right]^{2}$ and setting $\tau=e^{u / \epsilon^{2}}$ we obtain

$$
\begin{gathered}
\left(e^{\epsilon \triangle_{123} u+\triangle_{23} u+\triangle_{13} u+\triangle_{12} u}-e^{\triangle_{23} u}-e^{\triangle_{13} u}-e^{\triangle_{12} u}\right)^{2}= \\
4\left(e^{\triangle_{13} u+\triangle_{23} u}+e^{\triangle_{12} u+\triangle_{13} u}+e^{\triangle_{12} u+\triangle_{23} u}-e^{\epsilon \triangle_{123} u+\triangle_{23} u+\triangle_{13} u+\triangle_{12} u}-e^{\triangle_{23} u+\triangle_{13} u+\triangle_{12} u}\right) .
\end{gathered}
$$

Its dispersionless limit is

$$
\left(e^{u_{23}+u_{13}+u_{12}}-e^{u_{23}}-e^{u_{13}}-e^{u_{12}}\right)^{2}=4\left(e^{u_{13}+u_{23}}+e^{u_{12}+u_{13}}+e^{u_{12}+u_{23}}-2 e^{u_{23}+u_{13}+u_{12}}\right) .
$$

It is remarkable that this dispersionless equation decouples into the product of four dispersionless BKP-type equations: setting $u=2 v$ we obtain

$$
\begin{gathered}
\left(e^{v_{23}+v_{13}+v_{12}}+e^{v_{23}}+e^{v_{13}}+e^{v_{12}}\right)\left(e^{v_{23}+v_{13}+v_{12}}-e^{v_{23}}-e^{v_{13}}+e^{v_{12}}\right) \times \\
\left(e^{v_{23}+v_{13}+v_{12}}-e^{v_{23}}+e^{v_{13}}-e^{v_{12}}\right)\left(e^{v_{23}+v_{13}+v_{12}}+e^{v_{23}}-e^{v_{13}}-e^{v_{12}}\right)=0 .
\end{gathered}
$$

One can show that hydrodynamic reductions of each BKP-branch of the dispersionless equation are inherited by the full CKP equation. Multidimensional consistency of the CKP equation, interpreted as the Cayley hyperdeterminant, was established in [44, 12]. An alternative form of the CKP equation was proposed earlier in [22]. 


\section{Acknowledgements}

We thank Frank Nijhoff, Yuri Suris and Matteo Petrera for clarifying discussions. We also thank the referees for useful comments. The research of IR was supported by a scholarship from Loughborough University.

\section{References}

[1] V.E. Adler, The tangential map and associated integrable equations, J. Phys. A 42, no. 33 (2009) 332004, 12 pp.

[2] V.E. Adler, A.I. Bobenko and Yu.B. Suris, Classification of integrable equations on quadgraphs. The consistency approach, Comm. Math. Phys. 233, no. 3 (2003) 513-543.

[3] V.E. Adler, A.I. Bobenko and Yu.B. Suris, Classification of integrable discrete equations of octahedron type, Int. Math. Res. Not. no. 8 (2012) 1822-1889.

[4] R. Ball, M. Petrera and Yu.B. Suris, What is integrability of discrete variational systems arXiv:1307.0523v1.

[5] A.I. Bobenko and Yu.B. Suris, Integrable Systems on Quad-Graphs, Int. Math. Res. Not. no. 11 (2002) 573-611.

[6] L.V. Bogdanov and B.G. Konopelchenko, Analytic-bilinear approach to integrable hierarchies. II. Multicomponent KP and 2D Toda lattice hierarchies, J. Math. Phys. 39, no. 9 (1998) 4701-4728.

[7] L.V. Bogdanov and B.G. Konopelchenko, Möbius invariant integrable lattice equations associated with KP and 2DTL hierarchies, Phys. Lett. A 256, no. 1 (1999) 39-46.

[8] L.V. Bogdanov and B.G. Konopelchenko, On dispersionless BKP hierarchy and its reductions, J. Nonlinear Math. Phys. 12, suppl. 1 (2005) 64-73.

[9] P.A. Burovskii, E.V. Ferapontov and S.P. Tsarev, Second order quasilinear PDEs and conformal structures in projective space, International J. Math. 21, no. 6 (2010) 799-841.

[10] E. Date, M. Jimbo and T. Miwa, Journ. Phys. Soc. Japan 51 (1982) 4125.

[11] A. Doliwa, Lattice geometry of the Hirota equation. SIDE III-symmetries and integrability of difference equations (Sabaudia, 1998), 93-100, CRM Proc. Lecture Notes, 25, Amer. Math. Soc., Providence, RI, (2000).

[12] A. Doliwa, The C-(symmetric) quadrilateral lattice, its transformations and the algebrogeometric construction, J. Geom. Phys. 60, no. 5 (2010) 690-707.

[13] I.Ya. Dorfman and F. W. Nijhoff, On a $(2+1)$-dimensional version of the Krichever-Novikov equation, Physics Letters A 157, no. 2-3 (1991) 107-112.

[14] E.V. Ferapontov and K.R. Khusnutdinova, On integrability of (2+1)-dimensional quasilinear systems, Comm. Math. Phys. 248 (2004) 187-206. 
[15] E.V. Ferapontov and A. Moro, Dispersive deformations of hydrodynamic reductions of 2D dispersionless integrable systems, J. Phys. A: Math. Theor. 42 (2009) 035211, 15pp.

[16] E.V. Ferapontov, A. Moro and V.S. Novikov, Integrable equations in $2+1$ dimensions: deformations of dispersionless limits, J. Phys. A: Math. Theor. 42 (2009) 18pp.

[17] E.V. Ferapontov and J. Moss, Linearly degenerate PDEs and quadratic line complexes, arXiv:1204.2777, to appear in Comm. Anal. Geom. (2014).

[18] E.V. Ferapontov, V.S. Novikov and I. Roustemoglou, Towards the classification of integrable differential-difference equations in $2+1$ dimensions, J. Phys. A: Math. Theor. 46 (2013) 13pp.

[19] E.V. Ferapontov, B. Huard and A. Zhang, On the central quadric ansatz: integrable models and Painleve reductions, J. Phys. A: Math. Theor. 45 (2012) 195204.

[20] R. Hirota, Discrete analogue of a generalized Toda equation, J. Phys. Soc. Japan 50 (1981) $3785-3791$.

[21] B. Huard and V.S. Novikov, On classification of integrable Davey-Stewartson type equations, J. Phys. A 46, no. 27 (2013) 275202, 13 pp.

[22] R.M. Kashaev, On discrete three-dimensional equations associated with the local YangBaxter relation, Lett. Math. Phys. 35 (1996) 389-397.

[23] A.D. King and W.K. Schief, Tetrahedra, octahedra and cubo-octahedra: integrable geometry of multi-ratios, J. Phys. A 36, no. 3 (2003) 785-802.

[24] S. Klainerman, The null condition and global existence to nonlinear wave equations. Nonlinear systems of partial differential equations in applied mathematics, Part 1 (Santa Fe, N.M., 1984), 293-326, Lectures in Appl. Math. 23, Amer. Math. Soc., Providence, RI, 1986.

[25] C. Klein and K. Roidot, Numerical study of shock formation in the dispersionless Kadomtsev-Petviashvili equation and dispersive regularizations , Physica D, Vol. 265 (2013) $1-25$.

[26] C. Klein and K. Roidot, Numerical Study of the semiclassical limit of the Davey-Stewartson II equations, arXiv:1401.4745.

[27] B.G. Konopelchenko and W.K. Schief, Menelaus' theorem, Clifford configurations and inversive geometry of the Schwarzian KP hierarchy, J. Phys. A 35, no. 29 (2002) 6125-6144.

[28] B.G. Konopelchenko and W.K. Schief, Reciprocal figures, graphical statics, and inversive geometry of the Schwarzian BKP hierarchy, Stud. Appl. Math. 109, no. 2 (2002) 89-124.

[29] A. Kuniba, T. Nakanishi and J. Suzuki, T-systems and Y-systems in integrable systems, J. Phys. A: Math. Theor. 44 (2011) 103001 (146pp).

[30] I. Krichever, O. Lipan, P. Wiegmann and A. Zabrodin, Quantum integrable models and discrete classical Hirota equations, Commun. Math. Phys. 188 (1997) 267-304.

[31] D. Levi, L. Pilloni and P.M. Santini, Integrable three-dimensional lattices, J. Phys. A 14, no. 7 (1981) 1567-1575. 
[32] S. Lobb and F. Nijhoff, Lagrangian multiforms and multidimensional consistency, J. Phys. A 42, no. 45 (2009) 454013, 18 pp.

[33] S.B. Lobb, F.W. Nijhoff and G.R.W. Quispel, Lagrangian multiform structure for the lattice KP system, Journal of Physics A 42, no. 47 (2009) 472002 (11 pp).

[34] T. Miwa, On Hirota's difference equation, Proc. Japan Acad. 58 ser. A (1982) 9-12.

[35] F.W. Nijhoff, The direct linearizing transform for three-dimensional lattice equations, Physica 18D (1986) 380-381.

[36] F.W. Nijhoff, H.W. Capel, G.L. Wiersma and G.R.W. Quispel, Bäcklund transformations and three-dimensional lattice equations, Phys. Lett. A 105, no. 6 (1984) 267-272.

[37] F.W. Nijhoff and H.W. Capel, The direct linearization approach to hierarchies of integrable PDEs in $2+1$ dimensions. I. Lattice equations and the differential-difference hierarchies, Inverse Problems 6, no. 4 (1990) 567-590.

[38] F.W. Nijhoff and A.J. Walker, The discrete and continuous Painlevé VI hierarchy and the Garnier systems, Glasgow Mathematical Journal 43A (2001)109-123.

[39] J.J.C. Nimmo and W.K. Schief, Superposition principles associated with the Moutard transformation: an integrable discretization of a (2+1)-dimensional sine-Gordon system, Proc. R. Soc. Lond. A 453 (1997) 255-279, doi: 10.1098/rspa.1997.0015.

[40] J.J.C. Nimmo and W.K. Schief, An integrable discretization of a (2+1)-dimensional sineGordon equation, Stud. Appl. Math. 100, no. 3 (1998) 295-309.

[41] W.K. Schief, Lattice geometry of the discrete Darboux, KP, BKP and CKP equations. Menelaus' and Carnot's theorems, J. Nonlinear Math. Phys. 10, supp. 2 (2003) 194-208.

[42] K. Takasaki and T. Takebe, Integrable hierarchies and dispersionless limit, Rev. Math. Phys. 7, no. 5 (1995) 743-808.

[43] S.P. Tsarev and T. Wolf, Classification of three-dimensional integrable scalar discrete equations. Lett. Math. Phys. 84, no. 1 (2008) 31-39.

[44] S.P. Tsarev and T. Wolf, Hyperdeterminants as integrable discrete systems, J. Phys. A 42 , no. 45 (2009) 454023, 9 pp.

[45] G.L. Wiersma and H.W. Capel, Lattice equations, hierarchies and Hamiltonian structures: the Kadomtsev-Petviashvili equation, Phys. Lett. A 124, no. 3 (1987) 124-130.

[46] G.L. Wiersma and H.W. Capel, Lattice equations, hierarchies and Hamiltonian structures. II. KP-type of hierarchies on 2D lattices, Phys. A 149 (1988), no. 1-2, 4974.

[47] G.L. Wiersma and H.W. Capel, Lattice equations, hierarchies and Hamiltonian structures. III. The 2D Toda and KP hierarchies, Phys. A 149 (1988), no. 1-2, 75-106.

[48] A. Zabrodin, A survey of Hirota's difference equations, Theor. Math. Phys. 113 (1997) $1347-1392$. 